\title{
Physiological and Nutraceutical Quality of Green and Red Pigmented Lettuce in Response to $\mathrm{NaCl}$ Concentration in Two Successive Harvests
}

\author{
Petronia Carillo $^{1}\left(\right.$, Maria Giordano ${ }^{2} \odot$, Giampaolo Raimondi ${ }^{2}$, Francesco Napolitano ${ }^{2}$, \\ Emilio Di Stasio ${ }^{2}$, Marios C. Kyriacou ${ }^{3}$ (D) Maria Isabella Sifola ${ }^{1, *(\mathbb{D})}$ and Youssef Rouphael ${ }^{1, *(D)}$ \\ 1 Department of Environmental, Biological and Pharmaceutical Sciences and Technologies, \\ University of Campania "Luigi Vanvitelli", Via Vivaldi 43, 81100 Caserta, Italy; \\ petronia.carillo@unicampania.it \\ 2 Department of Agricultural Sciences, University of Naples Federico II, 80055 Portici, Italy; \\ maria.giordano@unina.it (M.G.); giampaolo.raimondi@unina.it (G.R.); \\ francesco.napolitano@hotmail.it (F.N.); emiliodistasio@gmail.com (E.D.S.) \\ 3 Department of Vegetable Crops, Agricultural Research Institute, Nicosia 1516, Cyprus; \\ m.kyriacou@ari.gov.cy \\ * Correspondence: sifola@unina.it (M.I.S.); youssef.rouphael@unina.it (Y.R.); Tel.: +39-081-2539-134 (Y.R.)
}

Received: 6 August 2020; Accepted: 8 September 2020; Published: 10 September 2020

\begin{abstract}
Nutritional eustress such as salinity or nutrient stress applied in soilless systems, is a convenient pre-harvest factor efficient in modulating the phytochemical components of horticultural crops, by triggering defensive mechanisms and accumulating plant secondary metabolites in plants tissues. Nevertheless, genetic material (cultivars with different pigmentation) dictates lettuce metabolites and physiological response to extrinsic eustress, with red leaf cultivars being highly nutrient packed notwithstanding the stress. Product quality can be meliorated equally by applying several cuts, a practice proven to increase bioactive compounds accumulation. In this study, we analyzed the effects of four salinity levels $(1,10,20$ and $30 \mathrm{mM} \mathrm{NaCl})$ on green and red pigmented Salad Bowl lettuce (Lactuca sativa L. var. acephala) in two successive harvests cultivated in a floating raft system. The morphological parameters, mineral composition, leaf gas exchanges, bioactive compounds, and antioxidant activity of both cultivars were assessed. The green cultivar exhibited superior crop productivity but was more prone to salinity effect than the red cultivar. Irrespective of cultivar and cut order, the net photosynthesis decreased with increasing salinity in the nutrient solution. The second cut incurred higher dry biomass, greater accumulation of most minerals and higher photosynthetic activity. In red lettuce, $20 \mathrm{mM} \mathrm{NaCl}$ proved adequate eustress to increase phytonutrients and beneficial minerals $(\mathrm{K}, \mathrm{Ca}$, and $\mathrm{Mg}$ ) with minimal loss of yield. Mild salinity and sequential harvest have proven effective pre-harvest tools in positively modulating the quality of lettuce. Eustress interaction with genotype was demonstrated as a promising field for future breeding programs targeting select genotypes for agronomic application of eustress to improve the nutraceutical value of vegetable crops.
\end{abstract}

Keywords: antioxidant activity; floating raft system; eustress; electrical conductivity; Lactuca sativa L.; leaf gas exchange; functional quality

\section{Introduction}

Lettuce (Lactuca sativa L.) is one of the main leafy green vegetables cultivated worldwide, whose consumption has steadily increased due to its perception as a health-promoting fresh food [1-3]. In particular, baby lettuce leaves with their petiole, at an optimum size of 8-12 cm long, have become 
very popular in recent years as minimally processed or fresh-cut salad vegetables ready to eat $[4,5]$. They represent an innovative produce corresponding to the lifestyle of modern consumers [2]. Indicatively, the sector of minimally processed agricultural products in Italy has grown from the early 1980 s to 2019 by about $400 \%$, with approximately 19.4 million regular consumers in 2017 [6]. In addition, Italy is the European leader of leafy green production with about 15,000 ha under greenhouse conditions located mainly in Campania, Veneto, and Lombardia regions [6].

Lettuce has a low calorific value and supplies a plethora of antioxidant compounds, such as vitamins $\mathrm{C}$ (ascorbic acid), E and folate (vitamin $\mathrm{B}_{9}$ ), chlorophylls, carotenoids and polyphenols, in addition to micro- and macro-nutrients and fiber [3,7-12]. There is growing evidence that these secondary bioactive metabolites, in particular polyphenols, can act as antioxidants counteracting the post-harvest decay of lettuce tissues and the development off-odors, and also as nutraceuticals with a preventive role against oxidative stress-related diseases, such as cardiovascular diseases, atherosclerosis, neurodegenerative disorders and cancer [3,13-16]. This evidence has attracted the interest of researchers and producers toward ways of enhancing the content of these compounds [3,13-16], including genetic modification and novel agronomic techniques [1]. However, EU policymakers have introduced very restrictive regulations concerning the use of genetically modified organisms, which consequently eliminates this tool from the array of breeders and producers [17]. Therefore, agronomic techniques such as cropping system, nutrient solution management and elicitation factors (e.g., salinity, high $\mathrm{CO}_{2}$, light intensity, and quality) are the favored methods employed to improve crop performance, to enhance the nutritional and nutraceutical qualities of products and extend their post-harvest life [14,18-21].

Recent studies on the application of mild stresses (i.e., salt or nutrient stress) to crop plants provide evidence to the existence of stress-related plasticity responsible for increased synthesis and accumulation of protective secondary metabolites that counteract stress [22-25]. These secondary metabolites, mostly deriving from phenylpropanoid and shikimate pathways, are able to scavenge reactive oxygen species (ROS), promote lignification and herbivore protection, and act as disease and stress signals [15]. For a positive stress (eustress) to increase the content of beneficial metabolites without significant detriment to plant growth and especially yield, a compromised level of stress must be found $[19,26]$. Salinity at an optimal mild dose (up to $6.0 \mathrm{dS} \mathrm{m}^{-1}$, equivalent to about $60 \mathrm{mM} \mathrm{NaCl}$ ) has been found to improve the content of ascorbic acid, $\alpha$-tocopherol and the antioxidant activity in Cichorium spinosum without significant yield reduction [27]. Neocleous et al. [28] also found that $10 \mathrm{mM}$ $\mathrm{NaCl}$ strongly increased ascorbic acid content in two baby lettuce cultivars (green "Paris Island" and red "Sanguine"). Leafy vegetable Amaranthus tricolor showed tremendous augmentation of carotenoids, ascorbic acid, total phenols, total flavonoids, phenolic acids, flavonoid compounds, and antioxidant activity at $100 \mathrm{mM} \mathrm{NaCl}$ stress with minimal yield reduction [29-31]. Furthermore, Colla et al. [32] found that by adding $30 \mathrm{mM}$ of sodium chloride $(\mathrm{NaCl})$ to the nutrient solution of cultured artichoke and cardoon, the leaf content of total phenolics, chlorogenic acid, cynarine and luteolin and the antioxidant activity increased. Long-term irrigation with $5 \mathrm{mM} \mathrm{NaCl}$ salt concentration was found to increase lutein $(+37 \%)$ and $\beta$-carotene $(+80 \%)$ in romaine lettuce without altering the visual quality or decreasing yield [15]. More recently, Rouphael et al. [18] showed, in a greenhouse study on green and red pigmented perilla, that the application of $10 \mathrm{mM} \mathrm{NaCl}$ enhanced the content of polyphenols while decreasing that of nitrate (an antinutrient); moreover, key aroma compounds increased, particularly in green perilla, following the application of $20 \mathrm{mM} \mathrm{NaCl}$ to the nutrient solution. The above evidence indeed highlights the motto of Paracelsus "dose makes the poison" [18].

Since the application of $\mathrm{NaCl}$ under soil conditions poses a high risk of plant overstress, soilless cultivation, particularly the floating raft system, may be an efficient tool to apply controlled eustress for enhancing plant secondary metabolism and sensory/functional product quality, provided the proper management of nutrient solution composition and concentration [4]. In addition, soilless cultivation presents numerous advantages over soil-based cultivation regardless of eustress application. In particular, (i) multiple cultivation cycles can be facilitated throughout the year; (ii) soil related pathogenic and abiotic stress conditions are alleviated; (iii) high density planting can be applied 
without weed control pressure; (iv) labor requirements are reduced; (v) products free of soil-particles and other debris can be readily harvested [24,33-36].

Another agronomic factor widely adopted for leafy greens that may modulate their secondary metabolic profile and nutraceutical quality could be sequential harvesting. The cut number in sequential harvesting may lead to physiological changes that affect plant metabolism. In a previous work on basil Nicoletto and co-workers [37] demonstrated that several harvests/cuts (3 or 4) of the apical part of the plant during the growth cycle may strongly enhance the phenolic content. Therefore, the application of sequential harvesting merits appraisal as a potential tool to modulate the nutraceutical quality of lettuce.

To our knowledge, the application of salinity as eustress in combination with successive harvests to modulate physiological and biochemical plant parameters has never been tested. Accordingly, in our study we analyzed the effects of four salinity levels $(1,10,20$ and $30 \mathrm{mM} \mathrm{NaCl})$ on green and red pigmented lettuce cultivars in two successive harvests with respect to morphological parameters, mineral composition, leaf gas exchange, bioactive compounds, and in vitro antioxidant activity.

\section{Materials and Methods}

\subsection{Growth Conditions, Lettuce Cultivars, Experimental Design and Salt Application}

The experimental work was carried out during the spring-summer 2015 growing cycle in a glasshouse at the experimental station 'Torre Lama Dipartimento di Agraria' (Bellizzi, Salerno $40^{\circ} 37^{\prime} 00^{\prime \prime}$ $\mathrm{N} 14^{\circ} 57^{\prime} 00^{\prime \prime} \mathrm{E}$ ). Two lettuce (Lactuca sativa $\mathrm{L}$. var. acephala) varieties with different leaf pigmentations: 'Green Salad Bowl' and 'Red Salad Bowl' (SAIS seed company, Cesena, Italy) were used as tested crop. The two lettuce varieties were sown on 22 March in polystyrene trays (190 holes) with a planting density of 1025 plants per square meter. Eleven days after sowing (DAS) lettuce plants were moved to a floating raft system. The system consisted of polystyrene plug trays, floating in wood tanks with a constant volume $(150 \mathrm{~L})$ of fresh nutrient solution. The dissolved oxygen concentration in the nutrient solution was always higher than $6 \mathrm{mg} \mathrm{L}^{-1}$. The experimental design was full factorial, with three factors: "cultivar" (CV) with two levels (i.e., green and red lettuce), "salinity" (S) four levels, namely 1, 10, 20, and $30 \mathrm{mM} \mathrm{NaCl}$ and "harvest" (cut 1 and cut 2). The concentration of the macro and microelement in the non-saline nutrient solution was: $12.0 \mathrm{mM}$ nitrate, $1.0 \mathrm{mM}$ ammonium, $1.75 \mathrm{mM}$ sulfur, $1.5 \mathrm{mM}$ phosphorus, $5.0 \mathrm{mM}$ potassium, $4.0 \mathrm{mM}$ calcium, $1.5 \mathrm{mM}$ magnesium, $1.0 \mathrm{mM}$ sodium, $1.0 \mathrm{mM}$ chloride, $20 \mu \mathrm{M}$ iron, $9 \mu \mathrm{M}$ manganese, $0.3 \mu \mathrm{M}$ copper, $1.6 \mu \mathrm{M}$ zinc, $20 \mu \mathrm{M}$ boron, and $0.3 \mu \mathrm{M}$ molybdenum, with an electrical conductivity (EC) of $1.9 \mathrm{dS} \mathrm{m}^{-1}$. The concentration of the macronutrients and micronutrients in the saline solutions were the same of the non-saline (NS) plus an additional 10, 20 and $30 \mathrm{mM} \mathrm{NaCl}$. The EC of the 10, 20 and $30 \mathrm{mM}$ solutions was 2.8, 4.0 and $5.1 \mathrm{dS} \mathrm{m}^{-1}$, respectively. The $\mathrm{pH}$ of the nutrient solution for all treatments was $6.0 \pm 0.2$. The 16 combinatorial treatments were arranged in a randomized complete-block design with three replicates for each cultivar, making a total of 48 experimental units (i.e., plots).

\subsection{Sample Preparation}

Determinations of total phenolic, antioxidant activity assays (lipophilic and hydrophilic fractions), mineral profile (nitrate, total nitrogen, phosphorus, potassium, calcium, magnesium, sodium, and chloride) were evaluated using lyophilized samples. Whereas, total ascorbic acid was done on liquid nitrogen quenched fresh biomass before being lyophilized.

\subsection{Leaf Area, Fresh Yield and Leaf Biomass Determination}

The red and green pigmented lettuce were harvested twice: 26 and 47 DAS, when the number of leaves reached approximately 5-6 leaves. In both harvest dates, the red and green pigmented lettuce were directly weighed, and total leaf area was quantified with an electronic area meter (LiCor 3100C model, LI-COR Biosciences, Lincoln, NE, USA). The lettuce fresh yield was recorded for each 
experimental unit and expressed in $\mathrm{kg}$ per square meter. The lettuce leaf tissues of the two varieties were dried in a forced-air oven at $70{ }^{\circ} \mathrm{C}$ for $72 \mathrm{~h}$ until constant weight to determine the dry leaf biomass. Leaf dry matter $(\mathrm{DM})$ percentage was calculated using the following formula: $100 \times$ dry leaf biomass/fresh weight.

\subsection{Physiological Parameters: SPAD Index and Leaf Gas Exchange}

Before the two harvests, the soil plant analysis development (SPAD) index and leaf gas exchange were determined. The SPAD index was quantified on 15 entirely (i.e., healthy) expanded lettuce leaves per experimental unit, by using a portable SPAD-502 chlorophyll meter (Konica Minolta, Tokyo, Japan). The following physiological measurements were determined by an LCA-4; ADC portable gas exchange analyzer (BioScientific Ltd., Hoddesdon, UK) equipped with a $6.25 \mathrm{~cm}^{2}$ broadleaf chamber: net carbon dioxide assimilation rate, stomatal resistance, and transpiration. The leaf gas exchange parameters were quantified on 12 entirely expanded lettuce leaves per treatment. Physiological (i.e., intrinsic) water use efficiency (WUE) was calculated as the ratio between net photosynthetic $\mathrm{CO}_{2}$ rate and transpiration.

\subsection{Macro and Micro Mineral Content Analysis}

The lettuce leaf tissues were analyzed for the following macroelements sodium and chloride: nitrogen $(\mathrm{N})$, nitrate $\left(\mathrm{NO}_{3}\right)$, phosphorus $(\mathrm{P})$, potassium $(\mathrm{K})$, calcium $(\mathrm{Ca})$, magnesium $(\mathrm{Mg}), \operatorname{sodium}(\mathrm{Na})$ and chloride $(\mathrm{Cl})$. One gram of lettuce ground material was used for the determination of total $\mathrm{N}$ using the micro-Kjeldahl method [38]. Moreover, $0.25 \mathrm{~g}$ ground material was also analyzed by ion chromatography (ICS-3000, Dionex, Sunnyvale, CA, USA) to determine the other minerals content according to the method proposed by Rouphael et al. [39]. The result of nitrate was expressed as $\mathrm{mg}$ $\mathrm{kg}^{-1}$ fresh weight (fw), while the other macrominerals, sodium and chloride were expressed as $\mathrm{g} \mathrm{kg}-1$ dry weight $(\mathrm{dw})$.

\subsection{Bioactive Compounds and Antioxidant Activity Assays Analysis}

The bioactive compounds (total ascorbic acid and total phenols) as well as antioxidant activity assays were assessed by spectrophotometric detection (model Hach DR 2000, Hach Co., Loveland, CO, USA). Total ascorbic acid and total phenols were assessed based on the method proposed by Sarker et al. [40] and Sarker and Oba [41] (i.e., Folin-Ciocalteau procedure), respectively. The absorbance of the solution was read at 525 and $765 \mathrm{~nm}$ for total ascorbic acid and total phenols, respectively.

For the determination of the hydrophilic and lipophilic antioxidant activity assays, N, N-dimethyl-p-phenylenediamine (DMPD) method [42] and 2,20-azinobis 3-ethylbenzothiazoline-6-sulfonic acid (ABTS) method [43], respectively, were adopted. Antioxidant capacities were measured by UV-Vis spectrophotometry, at $505 \mathrm{~nm}$, for the hydrophilic fraction, and at $734 \mathrm{~nm}$ for lipophilic fraction.

\subsection{Statistics}

The experimental data were analyzed by the three-way analysis of variance (Cultivar, Salinity and Harvest) using the SPSS software version 21, and the Duncan multiple range test was performed for the mean separations at 0.05 probability level. The loading plot and score plot of all agronomical, physiological and qualitative parameters of red and green pigmented baby lettuce were determined after principal component analysis (PCA) by using Minitab ${ }^{\circledR} 18$ statistical software (Minitab LLC, State College, PA, USA) [44].

\section{Results}

\subsection{Biometric Measurements}

With respect to the biometric variables, significant differences were observed between cultivars for fresh yield, leaf dry biomass and leaf dry matter (DM) content but not for leaf area (Table 1). 
Superior crop productivity, dry biomass and leaf DM content were observed in green lettuce (Table 1). $\mathrm{NaCl}$ treatments had significant effect on all the morphological parameters. The high $\mathrm{NaCl}$ treatment $(30 \mathrm{mM} \mathrm{NaCl})$ suppressed both leaf area and fresh yield, while an opposite trend was observed for leaf DM content. Moreover, the 1 and $10 \mathrm{mM} \mathrm{NaCl}$ treatments did not differ significantly from each other for leaf area, dry biomass, and leaf DM content (Table 1). Except for leaf area, cut order significantly affected all biometric variables examined: dry biomass and leaf DM content were higher at cut 2, at the expense of marketable fresh yield (Table 1). Finally, irrespective of salt stress treatment (cultivar $\times$ cut interaction), the percentage of leaf DM increase caused by cut order was significantly higher in green lettuce (by $14.9 \%)$, compared to red lettuce (11.7\%; Table 1 ).

Table 1. Significance of the main factors (cultivar, salinity and cut order) and their interactions on leaf area, total yield, leaf dry biomass and leaf dry matter percentage of Lactuca sativa L. var. acephala grown in a floating raft culture.

\begin{tabular}{|c|c|c|c|c|}
\hline Source of Variance & $\begin{array}{c}\text { Leaf Area } \\
\left(\mathrm{cm}^{2} \text { plant }^{-1}\right)\end{array}$ & $\begin{array}{l}\text { Fresh Yield } \\
\quad\left(\mathrm{kg} \mathrm{m}^{-2}\right)\end{array}$ & $\begin{array}{l}\text { Dry Biomass } \\
\qquad\left(\mathrm{g} \mathrm{m}^{-2}\right)\end{array}$ & $\begin{array}{l}\text { Leaf Dry Matter } \\
(\%)\end{array}$ \\
\hline \multicolumn{5}{|l|}{ Cultivar (CV) } \\
\hline Green & $92.10 \pm 2.55$ & $5.59 \pm 0.14 \mathrm{a}$ & $271.7 \pm 6.61 \mathrm{a}$ & $4.89 \pm 0.10 \mathrm{a}$ \\
\hline Red & $88.37 \pm 2.02$ & $4.98 \pm 0.13 b$ & $214.3 \pm 4.96 \mathrm{~b}$ & $4.33 \pm 0.07 b$ \\
\hline \multicolumn{5}{|l|}{ Salinity (S) } \\
\hline $1 \mathrm{mM} \mathrm{NaCl}$ & $99.58 \pm 3.16 \mathrm{a}$ & $5.82 \pm 0.22 \mathrm{a}$ & $261.7 \pm 14.6 \mathrm{a}$ & $4.48 \pm 0.13 b$ \\
\hline $10 \mathrm{mM} \mathrm{NaCl}$ & $94.92 \pm 2.67 \mathrm{a}$ & $5.39 \pm 0.16 b$ & $247.2 \pm 10.2 \mathrm{ab}$ & $4.58 \pm 0.12 b$ \\
\hline $20 \mathrm{mM} \mathrm{NaCl}$ & $88.25 \pm 1.69 \mathrm{~b}$ & $5.20 \pm 0.19 b$ & $235.1 \pm 10.3 \mathrm{~b}$ & $4.53 \pm 0.16 b$ \\
\hline $30 \mathrm{mM} \mathrm{NaCl}$ & $78.18 \pm 1.40 c$ & $4.73 \pm 0.15 c$ & $228.1 \pm 10.1 b$ & $4.83 \pm 0.16 \mathrm{a}$ \\
\hline \multicolumn{5}{|l|}{ Cut (C) } \\
\hline Cut 1 & $89.20 \pm 1.80$ & $5.49 \pm 0.14 \mathrm{a}$ & $234.9 \pm 7.15 b$ & $4.27 \pm 0.06 b$ \\
\hline Cut 2 & $91.27 \pm 2.74$ & $5.08 \pm 0.15 b$ & $251.1 \pm 9.12 \mathrm{a}$ & $4.94 \pm 0.10 \mathrm{a}$ \\
\hline \multicolumn{5}{|l|}{$\mathrm{CV} \times \mathrm{S}$} \\
\hline Green $\times 1 \mathrm{mM} \mathrm{NaCl}$ & $101.65 \pm 5.37$ & $6.15 \pm 0.34$ & $297.0 \pm 17.6$ & $4.83 \pm 0.13$ \\
\hline Green $\times 10 \mathrm{mM} \mathrm{NaCl}$ & $98.66 \pm 4.55$ & $5.72 \pm 0.17$ & $272.2 \pm 9.05$ & $4.77 \pm 0.15$ \\
\hline Green $\times 20 \mathrm{mM} \mathrm{NaCl}$ & $89.82 \pm 1.71$ & $5.47 \pm 0.30$ & $261.0 \pm 12.5$ & $4.81 \pm 0.25$ \\
\hline Green $\times 30 \mathrm{mM} \mathrm{NaCl}$ & $78.28 \pm 0.91$ & $5.02 \pm 0.12$ & $256.8 \pm 7.61$ & $5.14 \pm 0.26$ \\
\hline Red × $1 \mathrm{mM} \mathrm{NaCl}$ & $97.51 \pm 3.68$ & $5.48 \pm 0.21$ & $226.4 \pm 11.3$ & $4.13 \pm 0.10$ \\
\hline $\operatorname{Red} \times 10 \mathrm{mM} \mathrm{NaCl}$ & $91.19 \pm 2.25$ & $5.06 \pm 0.21$ & $222.2 \pm 11.3$ & $4.40 \pm 0.18$ \\
\hline Red $\times 20 \mathrm{mM} \mathrm{NaCl}$ & $86.68 \pm 2.94$ & $4.93 \pm 0.20$ & $209.2 \pm 6.66$ & $4.26 \pm 0.15$ \\
\hline $\begin{array}{c}\operatorname{Red} \times 30 \mathrm{mM} \mathrm{NaCl} \\
\mathrm{S} \times \mathrm{C}\end{array}$ & $78.08 \pm 2.79$ & $4.43 \pm 0.22$ & $199.4 \pm 7.92$ & $4.51 \pm 0.11$ \\
\hline $1 \mathrm{mM} \mathrm{NaCl} \times$ Cut 1 & $94.94 \pm 2.95$ & $5.86 \pm 0.34$ & $250.8 \pm 19.7$ & $4.26 \pm 0.15$ \\
\hline $10 \mathrm{mM} \mathrm{NaCl} \times$ Cut 1 & $92.51 \pm 4.24$ & $5.57 \pm 0.19$ & $236.5 \pm 12.1$ & $4.24 \pm 0.10$ \\
\hline $20 \mathrm{mM} \mathrm{NaCl} \times$ Cut 1 & $88.37 \pm 3.00$ & $5.49 \pm 0.33$ & $228.9 \pm 15.4$ & $4.16 \pm 0.09$ \\
\hline $30 \mathrm{mM} \mathrm{NaCl} \times$ Cut 1 & $80.98 \pm 1.35$ & $5.03 \pm 0.13$ & $223.4 \pm 9.31$ & $4.43 \pm 0.10$ \\
\hline $1 \mathrm{mM} \mathrm{NaCl} \times$ Cut 2 & $104.22 \pm 5.17$ & $5.77 \pm 0.30$ & $272.5 \pm 22.4$ & $4.70 \pm 0.18$ \\
\hline $10 \mathrm{mM} \mathrm{NaCl} \times$ Cut 2 & $97.34 \pm 3.32$ & $5.22 \pm 0.26$ & $257.8 \pm 16.4$ & $4.93 \pm 0.10$ \\
\hline $20 \mathrm{mM} \mathrm{NaCl} \times$ Cut 2 & $88.14 \pm 1.87$ & $4.91 \pm 0.10$ & $241.3 \pm 14.7$ & $4.90 \pm 0.22$ \\
\hline $\begin{array}{c}30 \mathrm{mM} \mathrm{NaCl} \times \text { Cut } 2 \\
\mathrm{CV} \times \mathrm{C}\end{array}$ & $75.38 \pm 1.91$ & $4.43 \pm 0.21$ & $232.8 \pm 18.9$ & $5.22 \pm 0.22$ \\
\hline Green $\times$ Cut 1 & $89.09 \pm 3.00$ & $5.81 \pm 0.20$ & $260.6 \pm 8.31$ & $4.49 \pm 0.05 b$ \\
\hline Green $\times$ Cut 2 & $95.11 \pm 4.06$ & $5.37 \pm 0.19$ & $282.8 \pm 9.54$ & $5.28 \pm 0.11 \mathrm{a}$ \\
\hline Red × Cut 1 & $89.31 \pm 2.15$ & $5.17 \pm 0.14$ & $209.2 \pm 4.98$ & $4.06 \pm 0.05 c$ \\
\hline Red $\times$ Cut 2 & $87.43 \pm 3.50$ & $4.78 \pm 0.20$ & $219.4 \pm 8.58$ & $4.60 \pm 0.07 b$ \\
\hline
\end{tabular}


Table 1. Cont.

\begin{tabular}{|c|c|c|c|c|}
\hline Source of Variance & $\begin{array}{c}\text { Leaf Area } \\
\left(\mathrm{cm}^{2} \text { plant }^{-1}\right)\end{array}$ & $\begin{array}{c}\text { Fresh Yield } \\
\left(\mathrm{kg} \mathrm{m}^{-2}\right)\end{array}$ & $\begin{array}{l}\text { Dry Biomass } \\
\qquad\left(\mathrm{g} \mathrm{m}^{-2}\right)\end{array}$ & $\begin{array}{c}\text { Leaf Dry Matter } \\
(\%)\end{array}$ \\
\hline \multicolumn{5}{|l|}{ Significance } \\
\hline Cultivar (CV) & ns & $* * *$ & $* * *$ & $* * *$ \\
\hline Salinity (S) & $* * *$ & $* * *$ & $* *$ & $* * *$ \\
\hline Cut (C) & ns & $* *$ & $*$ & $* * *$ \\
\hline $\mathrm{CV} \times \mathrm{S}$ & ns & ns & ns & ns \\
\hline $\mathrm{S} \times \mathrm{C}$ & ns & ns & ns & ns \\
\hline $\mathrm{CV} \times \mathrm{C}$ & ns & ns & ns & $*$ \\
\hline $\mathrm{CV} \times \mathrm{S} \times \mathrm{C}$ & ns & ns & ns & ns \\
\hline
\end{tabular}

$\mathrm{ns},{ }^{*}, * * * * * *$ Non-significant or significant at $p \leq 0.05,0.01$, and 0.001 , respectively. Different letters indicate statistically different groups ( $p<0.05$, Duncan's post hoc test following ANOVA; $n=3)$.

\subsection{Leaf Mineral Profile}

The cut order was the only factor with significant effect on all analyzed minerals (Table 2). Cultivar and cut were the only factors with a significant effect on leaf total $\mathrm{N}$, which was higher in the red cultivar than in the green and in the first cut than in the second one (Table 2). Similarly, to total N, phosphorus content was also higher in red lettuce than in green cultivar and in the first cut than in the second one. Increasing the $\mathrm{NaCl}$ concentration from 1 to $30 \mathrm{mM}$ in the nutrient solution incurred a significant decrease in the phosphorus content with no significant differences between 1 and $10 \mathrm{mM}$ $\mathrm{NaCl}$ treatments (Table 2). Potassium was significantly affected by cultivar $\times$ salinity and cultivar $\times$ cut interactions. In particular, in green lettuce the concentration of $\mathrm{K}$ in leaf tissue decreased as the salinity in the nutrient solution increased from 10 to $30 \mathrm{mM} \mathrm{NaCl}$ with the lowest values recorded at 20 and $30 \mathrm{mM}$; whereas, the concentration of $\mathrm{K}$ remained unchanged in red pigmented lettuce under salt stress conditions. Averaged over salt treatment levels, red lettuce was able to accumulate more potassium than the green lettuce $(+91 \%$ and $+30 \%$ in cut 1 and cut 2 , respectively) (Table 2$)$. The concentrations of bivalent cations $\mathrm{Ca}$ and $\mathrm{Mg}$ in leaf tissue were significantly affected by cultivar $\times$ salinity and cultivar $\times$ cut interactions (Table 2 ). When averaged over cut order (cultivar $\times$ salinity interaction), the percentage of $\mathrm{Ca}$ and $\mathrm{Mg}$ reduction in leaf tissue caused by salinity was significantly lower (17.0-35.1\% and $17.1-29.5 \%$ for $\mathrm{Ca}$ and $\mathrm{Mg}$, respectively) in the red lettuce cultivar compared to those recorded in the green pigmented variety $(13.8-53.8 \%$ and $18.4-44.0 \%$ for $\mathrm{Ca}$ and $\mathrm{Mg}$, respectively; Table 2). Moreover, irrespective of lettuce cultivar (salinity $\times$ cut interaction), the highest concentrations of $\mathrm{Ca}$ and $\mathrm{Mg}$ were recorded in the $1 \mathrm{mM}$ cut 2 treatment followed by lettuce plants fertigated with $10 \mathrm{mM} \mathrm{NaCl}$ and harvested 47 DAS (cut 2; Table 2).

The concentrations of both toxic ions $(\mathrm{Na}$ and $\mathrm{Cl}$ ) were not influenced by the cultivar $\times$ cut interaction, but by the cultivar $\times$ salinity and salinity $\times$ cut (only for Na) interactions (Table 2). The highest $\mathrm{Na}$ content was attained in response to the high $\mathrm{NaCl}$ treatment. The mean cultivar $\mathrm{Na}$ content increased by $45.1 \%$ at cut 2 relative to cut 1 ; however, a cultivar $\times$ cut interaction revealed that the increase incurred by the red lettuce was $266.7-687 \%$. On the other hand, in green lettuce a significant increase (330.1-662.8\%) was also noted depending on the $\mathrm{NaCl}$ concentration in the nutrient solution (Table 2). Similarly, to $\mathrm{Na}$, the highest $\mathrm{Cl}$ concentration was attained in response to the high $\mathrm{NaCl}$ treatment $(30 \mathrm{mM})$. The mean cultivar $\mathrm{Cl}$ content was higher in the red cultivar than in the green one; however a salinity $\times$ cut interaction revealed that the increase incurred by the red pigmented lettuce was significantly higher at cut 2 compared to cut 1 (Table 2). 
Table 2. Significance of the main factors (cultivar, salinity and cut order) and their interactions on leaf mineral composition (total nitrogen [N], phosphorus [P], potassium $[\mathrm{K}]$, calcium $[\mathrm{Ca}]$, magnesium $[\mathrm{Mg}]$, sodium $[\mathrm{Na}]$, chloride $[\mathrm{Cl}]$ ) of Lactuca sativa $\mathrm{L}$. var. acephala grown in a floating raft culture

\begin{tabular}{|c|c|c|c|c|c|c|c|}
\hline Source of Variance & $\underset{\left(\mathrm{g} \mathrm{kg}^{-1} \mathrm{dw}\right)}{\mathrm{N}}$ & $\begin{array}{c}P \\
\left(g_{k g}^{-1} \mathrm{dw}\right)\end{array}$ & $\begin{array}{c}K \\
\left(g_{k g}^{-1} \mathrm{dw}\right)\end{array}$ & $\begin{array}{c}\text { Ca } \\
\left(\mathrm{g} \mathrm{kg}^{-1} \mathrm{dw}\right)\end{array}$ & $\underset{\left(g_{k g}^{-1} \mathrm{dw}\right)}{\mathrm{Mg}}$ & $\begin{array}{c}\mathrm{Na} \\
\left(\mathrm{g} \mathrm{kg}^{-1} \mathrm{dw}\right)\end{array}$ & $\begin{array}{c}\mathrm{Cl} \\
\left(\mathrm{g} \mathrm{kg}^{-1} \mathrm{dw}\right)\end{array}$ \\
\hline \multicolumn{8}{|l|}{ Cultivar (CV) } \\
\hline Green & $40.51 \pm 0.32 b$ & $13.80 \pm 0.37 b$ & $38.11 \pm 2.13 b$ & $7.68 \pm 0.61 \mathrm{a}$ & $3.02 \pm 0.21$ & $14.89 \pm 1.78$ & $22.82 \pm 2.05 b$ \\
\hline Red & $41.54 \pm 0.52 \mathrm{a}$ & $14.63 \pm 0.24 \mathrm{a}$ & $59.61 \pm 0.94 \mathrm{a}$ & $6.75 \pm 0.37 b$ & $3.02 \pm 0.17$ & $13.23 \pm 1.67$ & $26.54 \pm 2.05 \mathrm{a}$ \\
\hline \multicolumn{8}{|l|}{ Salinity (S) } \\
\hline $1 \mathrm{mM} \mathrm{NaCl}$ & $40.72 \pm 0.52$ & $15.27 \pm 0.34 \mathrm{a}$ & $53.02 \pm 2.78 \mathrm{a}$ & $9.56 \pm 0.63 a$ & $3.87 \pm 0.32 \mathrm{a}$ & $2.98 \pm 0.29 \mathrm{~d}$ & $10.00 \pm 0.88 \mathrm{~d}$ \\
\hline $10 \mathrm{mM} \mathrm{NaCl}$ & $40.93 \pm 0.52$ & $14.56 \pm 0.34 \mathrm{ab}$ & $52.31 \pm 2.48 \mathrm{a}$ & $8.10 \pm 0.52 b$ & $3.18 \pm 0.19 b$ & $11.91 \pm 1.05 c$ & $23.43 \pm 0.88 c$ \\
\hline $20 \mathrm{mM} \mathrm{NaCl}$ & $40.94 \pm 0.92$ & $14.14 \pm 0.43 b$ & $45.91 \pm 4.69 \mathrm{~b}$ & $6.02 \pm 0.55 c$ & $2.60 \pm 0.21 c$ & $18.31 \pm 1.34 \mathrm{~b}$ & $30.01 \pm 0.94 b$ \\
\hline $30 \mathrm{mM} \mathrm{NaCl}$ & $41.53 \pm 0.49$ & $12.90 \pm 0.46 c$ & $44.21 \pm 4.83 \mathrm{~b}$ & $5.18 \pm 0.39 \mathrm{~d}$ & $2.42 \pm 0.10 c$ & $23.03 \pm 1.24 \mathrm{a}$ & $35.28 \pm 1.20 \mathrm{a}$ \\
\hline \multicolumn{8}{|l|}{ Cut (C) } \\
\hline Cut 1 & $42.16 \pm 0.31 \mathrm{a}$ & $15.00 \pm 0.28 \mathrm{a}$ & $47.83 \pm 3.60 b$ & $5.89 \pm 0.43 b$ & $2.49 \pm 0.13 b$ & $11.47 \pm 1.25 b$ & $24.09 \pm 1.71 b$ \\
\hline Cut 2 & $39.90 \pm 0.43 b$ & $13.43 \pm 0.29 b$ & $49.90 \pm 1.56 \mathrm{a}$ & $8.54 \pm 0.43 a$ & $3.54 \pm 0.18 a$ & $16.64 \pm 1.96 \mathrm{a}$ & $25.27 \pm 2.40 a$ \\
\hline \multicolumn{8}{|l|}{$\mathrm{CV} \times \mathrm{S}$} \\
\hline Green $\times 1 \mathrm{mM} \mathrm{NaCl}$ & $39.55 \pm 0.66$ & $15.19 \pm 0.58$ & $44.48 \pm 2.05 b$ & $10.74 \pm 0.73 a$ & $4.12 \pm 0.39 \mathrm{a}$ & $3.15 \pm 0.52 \mathrm{e}$ & $8.45 \pm 0.70$ \\
\hline Green $\times 10 \mathrm{mM} \mathrm{NaCl}$ & $40.72 \pm 0.54$ & $13.99 \pm 0.51$ & $45.26 \pm 1.69 b$ & $9.25 \pm 0.48 \mathrm{ab}$ & $3.36 \pm 0.24 \mathrm{abc}$ & $13.55 \pm 1.17 \mathrm{~cd}$ & $21.33 \pm 0.90$ \\
\hline Green $\times 20 \mathrm{mM} \mathrm{NaCl}$ & $41.01 \pm 0.67$ & $14.01 \pm 0.75$ & $32.32 \pm 4.43 c$ & $5.77 \pm 1.00 \mathrm{~d}$ & $2.29 \pm 0.37 \mathrm{~d}$ & $18.82 \pm 2.18 b$ & $27.99 \pm 1.42$ \\
\hline Green $\times 30 \mathrm{mM} \mathrm{NaCl}$ & $40.76 \pm 0.68$ & $12.02 \pm 0.61$ & $30.38 \pm 4.44 c$ & $4.96 \pm 0.69 \mathrm{~d}$ & $2.29 \pm 0.14 \mathrm{~d}$ & $24.03 \pm 1.99 \mathrm{a}$ & $33.51 \pm 2.03$ \\
\hline Red × $1 \mathrm{mM} \mathrm{NaCl}$ & $41.88 \pm 0.46$ & $15.34 \pm 0.40$ & $61.57 \pm 0.85 a$ & $8.38 \pm 0.82 b c$ & $3.62 \pm 0.52 a b$ & $2.80 \pm 0.29 \mathrm{e}$ & $11.54 \pm 1.39$ \\
\hline Red $\times 10 \mathrm{mM} \mathrm{NaCl}$ & $41.13 \pm 0.93$ & $15.13 \pm 0.36$ & $59.36 \pm 2.08 \mathrm{a}$ & $6.95 \pm 0.66 \mathrm{~cd}$ & $3.00 \pm 0.30 \mathrm{bcd}$ & $10.27 \pm 1.55 \mathrm{~d}$ & $25.53 \pm 0.92$ \\
\hline Red $\times 20 \mathrm{mM} \mathrm{NaCl}$ & $40.86 \pm 1.80$ & $14.26 \pm 0.47$ & $59.49 \pm 1.78 \mathrm{a}$ & $6.26 \pm 0.55 \mathrm{~d}$ & $2.90 \pm 0.15 \mathrm{bcd}$ & $17.80 \pm 1.76 \mathrm{bc}$ & $32.04 \pm 0.52$ \\
\hline $\begin{array}{c}\operatorname{Red} \times 30 \mathrm{mM} \mathrm{NaCl} \\
\mathrm{S} \times \mathrm{C}\end{array}$ & $42.29 \pm 0.60$ & $13.77 \pm 0.51$ & $58.03 \pm 2.57 \mathrm{a}$ & $5.40 \pm 0.43 \mathrm{~d}$ & $2.55 \pm 0.13 \mathrm{~cd}$ & $22.04 \pm 1.55 \mathrm{ab}$ & $37.05 \pm 0.96$ \\
\hline $1 \mathrm{mM} \mathrm{NaCl} \times$ Cut 1 & $41.36 \pm 0.64$ & $16.15 \pm 0.28$ & $52.91 \pm 4.70$ & $7.95 \pm 0.70 b c$ & $2.92 \pm 0.26 c$ & $3.56 \pm 0.38 \mathrm{f}$ & $12.12 \pm 1.13 \mathrm{e}$ \\
\hline $10 \mathrm{mM} \mathrm{NaCl} \times$ Cut 1 & $41.91 \pm 0.47$ & $15.26 \pm 0.22$ & $53.78 \pm 4.36$ & $6.96 \pm 0.69 \mathrm{~cd}$ & $2.62 \pm 0.15 \mathrm{~cd}$ & $9.06 \pm 1.08 \mathrm{e}$ & $23.68 \pm 1.66 \mathrm{~d}$ \\
\hline $20 \mathrm{mM} \mathrm{NaCl} \times$ Cut 1 & $42.99 \pm 0.75$ & $14.91 \pm 0.53$ & $42.82 \pm 9.09$ & $4.32 \pm 0.35 \mathrm{e}$ & $2.14 \pm 0.32 \mathrm{~d}$ & $14.07 \pm 0.67 \mathrm{~d}$ & $28.33 \pm 1.51 c$ \\
\hline $30 \mathrm{mM} \mathrm{NaCl} \times$ Cut 1 & $42.38 \pm 0.53$ & $13.66 \pm 0.58$ & $41.80 \pm 9.47$ & $4.32 \pm 0.57 \mathrm{e}$ & $2.28 \pm 0.17 \mathrm{~d}$ & $19.19 \pm 0.38 c$ & $32.22 \pm 1.51 \mathrm{~b}$ \\
\hline $1 \mathrm{mM} \mathrm{NaCl} \times$ Cut 2 & $40.07 \pm 0.78$ & $14.38 \pm 0.33$ & $53.14 \pm 3.47$ & $11.16 \pm 0.50 \mathrm{a}$ & $4.82 \pm 0.12 \mathrm{a}$ & $2.39 \pm 0.29 f$ & $7.87 \pm 0.53 \mathrm{f}$ \\
\hline $10 \mathrm{mM} \mathrm{NaCl} \times$ Cut 2 & $39.94 \pm 0.75$ & $13.87 \pm 0.52$ & $50.84 \pm 2.68$ & $9.24 \pm 0.46 b$ & $3.74 \pm 0.09 \mathrm{~b}$ & $14.75 \pm 0.66 \mathrm{~d}$ & $23.18 \pm 0.81 \mathrm{~d}$ \\
\hline $20 \mathrm{mM} \mathrm{NaCl} \times$ Cut 2 & $38.89 \pm 1.20$ & $13.36 \pm 0.52$ & $48.99 \pm 3.18$ & $7.71 \pm 0.23 c$ & $3.05 \pm 0.10 c$ & $22.55 \pm 0.56 b$ & $31.69 \pm 0.70 \mathrm{~b}$ \\
\hline $30 \mathrm{mM} \mathrm{NaCl} \times$ Cut 2 & $40.68 \pm 0.70$ & $12.13 \pm 0.60$ & $46.62 \pm 3.27$ & $6.05 \pm 0.23 \mathrm{~d}$ & $2.56 \pm 0.08 \mathrm{~cd}$ & $26.88 \pm 0.85 a$ & $38.34 \pm 0.53 a$ \\
\hline
\end{tabular}


Table 2. Cont

\begin{tabular}{|c|c|c|c|c|c|c|c|}
\hline Source of Variance & $\frac{N}{\left(\mathrm{~g} \mathrm{~kg}^{-1} \mathrm{dw}\right)}$ & $\begin{array}{c}P \\
\left(\mathrm{~g} \mathrm{~kg}^{-1} \mathrm{dw}\right)\end{array}$ & $\begin{array}{c}\mathrm{K} \\
\left(\mathrm{g} \mathrm{kg}^{-1} \mathrm{dw}\right)\end{array}$ & $\begin{array}{c}\mathrm{Ca} \\
\left(\mathrm{g} \mathrm{kg}^{-1} \mathrm{dw}\right)\end{array}$ & $\underset{\left(\mathrm{g} \mathrm{kg}^{-1} \mathrm{dw}\right)}{\mathrm{Mg}}$ & $\begin{array}{c}\mathrm{Na} \\
\left(\mathrm{g} \mathrm{kg}^{-1} \mathrm{dw}\right)\end{array}$ & $\begin{array}{c}\mathrm{Cl} \\
\left(\mathrm{g} \mathrm{kg}^{-1} \mathrm{dw}\right)\end{array}$ \\
\hline \multicolumn{8}{|l|}{$\mathrm{CV} \times \mathrm{C}$} \\
\hline Green $\times$ Cut 1 & $41.43 \pm 0.34$ & $15.01 \pm 0.40 \mathrm{a}$ & $32.84 \pm 3.53 \mathrm{~d}$ & $6.19 \pm 0.82$ & $2.48 \pm 0.23$ & $12.27 \pm 1.70$ & $21.20 \pm 2.24$ \\
\hline Green $\times$ Cut 2 & $39.60 \pm 0.40$ & $12.59 \pm 0.40 b$ & $43.39 \pm 1.19 c$ & $9.17 \pm 0.69$ & $3.55 \pm 0.29$ & $17.51 \pm 3.01$ & $24.43 \pm 3.48$ \\
\hline Red $\times$ Cut 1 & $42.89 \pm 0.43$ & $14.98 \pm 0.40 \mathrm{a}$ & $62.81 \pm 0.88 \mathrm{a}$ & $5.58 \pm 0.32$ & $2.50 \pm 0.11$ & $10.67 \pm 1.88$ & $26.98 \pm 2.40$ \\
\hline Red $\times$ Cut 2 & $40.20 \pm 0.78$ & $14.28 \pm 0.26 a$ & $56.41 \pm 1.01 \mathrm{~b}$ & $7.92 \pm 0.48$ & $3.54 \pm 0.23$ & $15.78 \pm 2.63$ & $26.10 \pm 3.43$ \\
\hline \multicolumn{8}{|l|}{ Significance } \\
\hline Cultivar (CV) & $*$ & $* *$ & $* * *$ & $* * *$ & ns & ns & $* * *$ \\
\hline Salinity (S) & ns & $* * *$ & $* * *$ & $* * *$ & $* * *$ & $* * *$ & $* * *$ \\
\hline Cut (C) & $* * *$ & $* * *$ & $*$ & $* * *$ & $* * *$ & $* * *$ & $*$ \\
\hline $\mathrm{CV} \times \mathrm{S}$ & ns & ns & $* * *$ & $* * *$ & $* * *$ & $*$ & ns \\
\hline $\mathrm{S} \times \mathrm{C}$ & ns & ns & ns & $* *$ & $* * *$ & $* * *$ & $* * *$ \\
\hline $\mathrm{CV} \times \mathrm{C}$ & ns & $* * *$ & $* * *$ & ns & ns & ns & ns \\
\hline $\mathrm{CV} \times \mathrm{S} \times \mathrm{C}$ & ns & ns & $* * *$ & $* *$ & ns & ns & ns \\
\hline
\end{tabular}

$\mathrm{ns},{ }^{*}, * *, * * *$ Non-significant or significant at $p \leq 0.05,0.01$, and 0.001 , respectively. Different letters indicate statistically different groups $(p<0.05$, Duncan's post hoc test following ANOVA; $n=3$ ). 


\subsection{SPAD Index and Leaf Gas Exchange}

The SPAD index was significantly affected by cultivar, salinity and cut order but not by their interactions (Table 3). The SPAD index values were higher in the red cultivar than in the green one and in the first cut than in the second one. Increasing the $\mathrm{NaCl}$ concentration in the nutrient solution reduced the SPAD index with the lowest values recorded with $30 \mathrm{mM} \mathrm{NaCl}$ (Table 3).

Table 3. Significance of the main factors (cultivar, salinity and cut order) and their interactions on SPAD index, net photosynthetic $\mathrm{CO}_{2}$ assimilation rate $\left[\mathrm{A}_{\mathrm{CO} 2}\right]$, stomatal resistance $\left[\mathrm{r}_{\mathrm{S}}\right]$, transpiration $[\mathrm{T}]$ and intrinsic water use efficiency $\left[\mathrm{WUE}_{\mathrm{i}}\right.$ ] of Lactuca sativa $\mathrm{L}$. var. acephala grown in a floating raft culture.

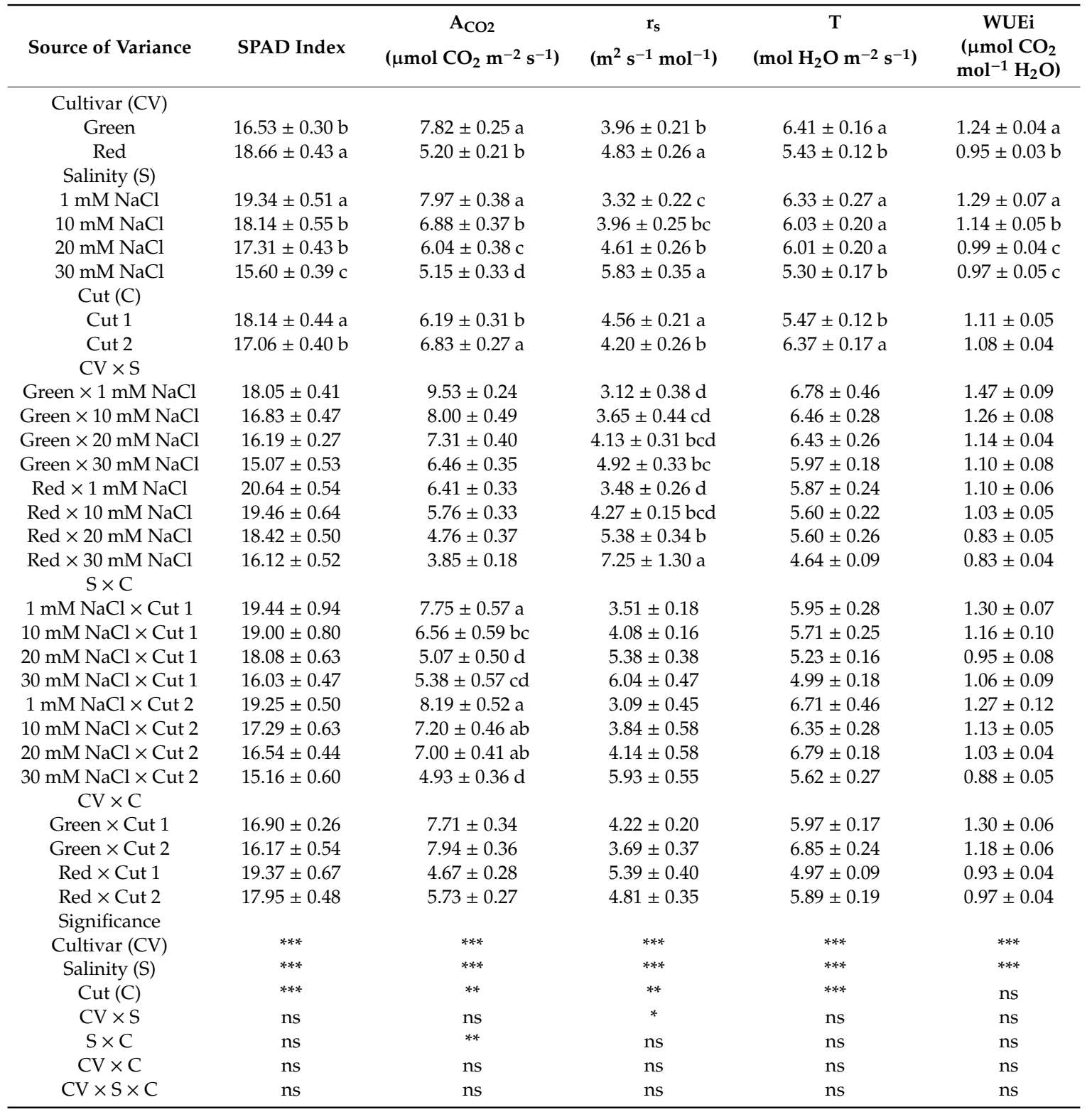

$\mathrm{ns}, * * * * * * *$ Non-significant or significant at $p \leq 0.05,0.01$, and 0.001 , respectively. Different letters indicate statistically different groups $(p<0.05$, Duncan's post hoc test following ANOVA; $n=3)$.

The net $\mathrm{CO}_{2}$ assimilation rate $\left(\mathrm{A}_{\mathrm{CO} 2}\right)$ content incurred a significant effect with respect to cultivar, as it was higher in green lettuce than in red one. Moreover, irrespective of cultivar and cut order, the $\mathrm{A}_{\mathrm{CO} 2}$ decreased linearly with increasing salinity in the nutrient solution, with the lowest values observed on plants treated with $30 \mathrm{mM} \mathrm{NaCl}$ (Table 3). Contrarily to $\mathrm{A}_{\mathrm{CO} 2}$, the stomatal resistance $\left(\mathrm{r}_{\mathrm{s}}\right)$ was significantly higher in red cultivar than in the green and in the first cut than in the second 
one. In addition, the $\mathrm{r}_{\mathrm{s}}$ increased linearly with increasing salinity in the nutrient solution, with a strong significant increase in the red cultivar at 20 and $30 \mathrm{mM} \mathrm{NaCl}$ compared to the green one at the same $\mathrm{NaCl}$ concentrations. However, no significant differences were found between cut 1 and cut 2 , independently of salinity (Table 3). Moreover, a significant effect of the genetic material was observed on both transpiration $(\mathrm{T})$ and intrinsic water use efficiency $\left(\mathrm{WUE}_{\mathrm{i}}\right)$. Transpiration and $\mathrm{WUE}_{\mathrm{i}}$ were higher by $18.4 \%$ and $30.5 \%$ in the green cultivar than in the red one (Table 3 ). Finally, increasing the salinity in the nutrient solution from 1 to $30 \mathrm{mM}$ suppressed $E$ only under severe salt stress $(30 \mathrm{mM})$, while the reduction in $\mathrm{WUE}_{\mathrm{i}}$ initiated directly after the addition of the mild salt stress $(10 \mathrm{mM} \mathrm{NaCl}$; Table 3).

\subsection{Qualitative Characteristics}

Except for the nitrate and hydrophilic antioxidant activity (HAA), the lipophilic antioxidant activity (LAA) and bioactive compounds such as total phenols (TP) and total ascorbic acid (TAA) were higher in red than in green baby lettuce (Table 4). Irrespective of the genetic material and cut order, reduced nitrate content was apparent only under severe stress conditions $(30 \mathrm{mM} \mathrm{NaCl})$. Both antioxidants molecules (TP and TAA) were significantly affected by cultivar $\times$ salinity, salinity $\times$ cut order and cultivar $\times$ cut order interactions (Table 4). For instance, the high significant interaction between cultivar and salinity was evident in our study, in particular the synthesis and accumulation of TP and TAA was not observed in green lettuce, while in red lettuce it peaked at $10 \mathrm{mM} \mathrm{NaCl}$ (for TP) and at $20 \mathrm{mM} \mathrm{NaCl}$ (for TAA; Table 4). Finally, the increase in TAA content was more pronounced in the red cultivar (Table 4).

Table 4. Significance of the main factors (cultivar, salinity and cut order) and their interactions on nitrate content, lipophilic antioxidant activity (LAA), hydrophilic antioxidant activity (HAA), total phenols (TP) and total ascorbic acid (TAA) of Lactuca sativa L. var. acephala grown in a floating raft culture.

\begin{tabular}{|c|c|c|c|c|c|}
\hline Source of Variance & $\begin{array}{c}\text { Nitrate } \\
\left(\mathrm{mg} \mathrm{kg}^{-1} \mathrm{fw}\right)\end{array}$ & $\begin{array}{c}\text { LAA } \\
\text { (mmol Trolox } \\
\left.100 \mathrm{~g}^{-1} \mathrm{dw}\right)\end{array}$ & $\begin{array}{c}\text { HAA } \\
\text { (mmol Ascorbic ac. } \\
\left.\text { eq. } \mathrm{kg}^{-1} \mathrm{dw}\right)\end{array}$ & $\begin{array}{c}\text { TP } \\
\text { (mg eq. Gallic } \\
\text { acid } \mathrm{g}^{-1} \mathrm{dw} \text { ) }\end{array}$ & $\begin{array}{c}\text { TAA } \\
\left(\mathrm{mg} 100 \mathrm{~g}^{-1} \mathrm{fw}\right)\end{array}$ \\
\hline \multicolumn{6}{|l|}{ Cultivar (CV) } \\
\hline Green & $2019 \pm 93.2$ & $5.05 \pm 0.22 b$ & $1.44 \pm 0.04$ & $51.10 \pm 1.99 \mathrm{~b}$ & $21.72 \pm 1.24 b$ \\
\hline Red & $1881 \pm 67.4$ & $6.73 \pm 0.30 a$ & $1.49 \pm 0.03$ & $66.50 \pm 2.89 \mathrm{a}$ & $30.61 \pm 2.54 \mathrm{a}$ \\
\hline \multicolumn{6}{|l|}{ Salinity (S) } \\
\hline $1 \mathrm{mM} \mathrm{NaCl}$ & $2125 \pm 142 \mathrm{a}$ & $5.81 \pm 0.48$ & $1.43 \pm 0.05$ & $58.88 \pm 4.19$ & $20.39 \pm 1.32 c$ \\
\hline $10 \mathrm{mM} \mathrm{NaCl}$ & $2048 \pm 120 \mathrm{a}$ & $6.07 \pm 0.48$ & $1.44 \pm 0.04$ & $58.45 \pm 5.59$ & $27.28 \pm 2.59 b$ \\
\hline $20 \mathrm{mM} \mathrm{NaCl}$ & $1925 \pm 91.4 \mathrm{a}$ & $5.82 \pm 0.38$ & $1.51 \pm 0.05$ & $59.88 \pm 3.88$ & $35.54 \pm 3.91 \mathrm{a}$ \\
\hline $30 \mathrm{mM} \mathrm{NaCl}$ & $1703 \pm 69.3 b$ & $5.85 \pm 0.47$ & $1.47 \pm 0.06$ & $57.98 \pm 2.99$ & $21.44 \pm 1.89 \mathrm{c}$ \\
\hline \multicolumn{6}{|l|}{ Cut (C) } \\
\hline Cut 1 & $1679 \pm 39.5 b$ & $6.81 \pm 0.25 a$ & $1.58 \pm 0.02 \mathrm{a}$ & $67.10 \pm 2.80 \mathrm{a}$ & $24.43 \pm 1.81 \mathrm{~b}$ \\
\hline Cut 2 & $2221 \pm 75.3 \mathrm{a}$ & $4.97 \pm 0.25 b$ & $1.34 \pm 0.03 b$ & $50.50 \pm 1.90 \mathrm{~b}$ & $27.89 \pm 2.48 \mathrm{a}$ \\
\hline \multicolumn{6}{|l|}{$\mathrm{CV} \times \mathrm{S}$} \\
\hline Green $\times 1 \mathrm{mM} \mathrm{NaCl}$ & $2265 \pm 253$ & $4.71 \pm 0.51$ & $1.41 \pm 0.08$ & $54.11 \pm 2.81 \mathrm{bcd}$ & $21.10 \pm 2.02 c$ \\
\hline Green $\times 10 \mathrm{mM} \mathrm{NaCl}$ & $2154 \pm 185$ & $5.08 \pm 0.48$ & $1.45 \pm 0.05$ & $46.26 \pm 5.09 \mathrm{~d}$ & $22.32 \pm 3.00 \mathrm{c}$ \\
\hline Green $\times 20 \mathrm{mM} \mathrm{NaCl}$ & $1957 \pm 115$ & $5.25 \pm 0.52$ & $1.47 \pm 0.08$ & $51.07 \pm 4.18 \mathrm{~cd}$ & $23.72 \pm 1.82 c$ \\
\hline Green $\times 30 \mathrm{mM} \mathrm{NaCl}$ & $1700 \pm 108$ & $5.16 \pm 0.34$ & $1.43 \pm 0.09$ & $52.97 \pm 3.75 \mathrm{bcd}$ & $19.74 \pm 3.19 c$ \\
\hline Red × $1 \mathrm{mM} \mathrm{NaCl}$ & $1985 \pm 132$ & $6.91 \pm 0.54$ & $1.45 \pm 0.06$ & $63.66 \pm 7.76 \mathrm{ab}$ & $19.67 \pm 1.84 c$ \\
\hline Red $\times 10 \mathrm{mM} \mathrm{NaCl}$ & $1943 \pm 156$ & $7.05 \pm 0.6399$ & $1.44 \pm 0.07$ & $70.65 \pm 7.21 \mathrm{a}$ & $32.24 \pm 3.28 b$ \\
\hline $\mathrm{Red} \times 20 \mathrm{mM} \mathrm{NaCl}$ & $1892 \pm 152$ & $6.39 \pm 0.49$ & $1.54 \pm 0.08$ & $68.69 \pm 4.22 \mathrm{a}$ & $47.36 \pm 2.86 \mathrm{a}$ \\
\hline $\operatorname{Red} \times 30 \mathrm{mM} \mathrm{NaCl}$ & $1705 \pm 97.7$ & $6.55 \pm 0.81$ & $1.52 \pm 0.08$ & $63.00 \pm 3.91 \mathrm{ab}$ & $23.14 \pm 2.10 \mathrm{c}$ \\
\hline
\end{tabular}


Table 4. Cont.

\begin{tabular}{|c|c|c|c|c|c|}
\hline Source of Variance & $\begin{array}{c}\text { Nitrate } \\
\left(\mathrm{mg} \mathrm{kg}^{-1} \mathrm{fw}\right)\end{array}$ & $\begin{array}{c}\text { LAA } \\
(\mathrm{mmol} \text { Trolox } \\
\left.100 \mathrm{~g}^{-1} \mathrm{dw}\right)\end{array}$ & $\begin{array}{c}\text { HAA } \\
\text { (mmol Ascorbic ac. } \\
\left.\text { eq. } \mathrm{kg}^{-1} \mathrm{dw}\right)\end{array}$ & $\begin{array}{c}\text { TP } \\
\text { (mg eq. Gallic } \\
\text { acid } g^{-1} \text { dw) }\end{array}$ & $\begin{array}{c}\text { TAA } \\
\left(\mathrm{mg} 100 \mathrm{~g}^{-1} \mathrm{fw}\right)\end{array}$ \\
\hline \multicolumn{6}{|l|}{$\mathrm{S} \times \mathrm{C}$} \\
\hline $1 \mathrm{mM} \mathrm{NaCl} \times$ Cut 1 & $1806 \pm 66.2$ & $6.75 \pm 0.59$ & $1.56 \pm 0.03$ & $68.56 \pm 5.91 \mathrm{ab}$ & $19.02 \pm 1.04 \mathrm{c}$ \\
\hline $10 \mathrm{mM} \mathrm{NaCl} \times$ Cut 1 & $1733 \pm 91.8$ & $6.87 \pm 0.48$ & $1.53 \pm 0.06$ & $70.96 \pm 7.13 \mathrm{a}$ & $21.15 \pm 2.53 c$ \\
\hline $20 \mathrm{mM} \mathrm{NaCl} \times$ Cut 1 & $1682 \pm 66.6$ & $6.76 \pm 0.38$ & $1.63 \pm 0.05$ & $66.46 \pm 5.54 \mathrm{ab}$ & $33.75 \pm 4.69 \mathrm{ab}$ \\
\hline $30 \mathrm{mM} \mathrm{NaCl} \times$ Cut 1 & $1494 \pm 28.2$ & $6.85 \pm 0.64$ & $1.62 \pm 0.04$ & $62.42 \pm 4.51 \mathrm{abc}$ & $23.81 \pm 2.32 b c$ \\
\hline $1 \mathrm{mM} \mathrm{NaCl} \times$ Cut 2 & $2443 \pm 210$ & $4.87 \pm 0.57$ & $1.30 \pm 0.05$ & $49.21 \pm 2.23 \mathrm{~cd}$ & $21.75 \pm 2.41 \mathrm{c}$ \\
\hline $10 \mathrm{mM} \mathrm{NaCl} \times$ Cut 2 & $2364 \pm 122$ & $5.27 \pm 0.72$ & $1.36 \pm 0.02$ & $45.95 \pm 4.90 \mathrm{~d}$ & $33.41 \pm 2.86 \mathrm{ab}$ \\
\hline $20 \mathrm{mM} \mathrm{NaCl} \times$ Cut 2 & $2167 \pm 94.0$ & $4.87 \pm 0.38$ & $1.39 \pm 0.07$ & $53.31 \pm 4.29 \mathrm{bcd}$ & $37.33 \pm 6.65 \mathrm{a}$ \\
\hline \multicolumn{6}{|l|}{$\mathrm{CV} \times \mathrm{C}$} \\
\hline Green $\times$ Cut 1 & $1737 \pm 52.9$ & $5.88 \pm 0.20$ & $1.56 \pm 0.03$ & $56.95 \pm 2.07 \mathrm{~b}$ & $21.42 \pm 1.54 \mathrm{c}$ \\
\hline Green $\times$ Cut 2 & $2301 \pm 138$ & $4.21 \pm 0.20$ & $1.32 \pm 0.04$ & $45.25 \pm 2.45 c$ & $22.03 \pm 2.00 \mathrm{c}$ \\
\hline Red × Cut 1 & $1620 \pm 55.8$ & $7.73 \pm 0.25$ & $1.61 \pm 0.04$ & $77.24 \pm 3.14 \mathrm{a}$ & $27.45 \pm 3.11 b$ \\
\hline Red $\times$ Cut 2 & $2142 \pm 59.1$ & $5.72 \pm 0.35$ & $1.36 \pm 0.03$ & $55.76 \pm 2.03 \mathrm{~b}$ & $33.76 \pm 3.93 \mathrm{a}$ \\
\hline \multicolumn{6}{|l|}{ Significance } \\
\hline Cultivar (CV) & ns & $* * *$ & ns & $* * *$ & $* * *$ \\
\hline Salinity (S) & $* *$ & ns & ns & ns & $* * *$ \\
\hline Cut (C) & $* * *$ & $* * *$ & $* * *$ & $* * *$ & * \\
\hline $\mathrm{CV} \times \mathrm{S}$ & ns & ns & ns & $*$ & $* * *$ \\
\hline $\mathrm{S} \times \mathrm{C}$ & ns & ns & ns & * & $* * *$ \\
\hline $\mathrm{CV} \times \mathrm{C}$ & ns & ns & ns & * & $*$ \\
\hline $\mathrm{CV} \times \mathrm{S} \times \mathrm{C}$ & ns & ns & ns & ns & ns \\
\hline
\end{tabular}

$\mathrm{ns},{ }^{*},{ }^{* *},{ }^{* * *}$ Non-significant or significant at $p \leq 0.05,0.01$, and 0.001 , respectively. Different letters indicate statistically different groups ( $p<0.05$, Duncan's post hoc test following ANOVA; $n=3)$.

\subsection{Principal Component Analysis (PCA)}

A principal component analysis was performed on all analyzed data, and the loading plot and scores are reported in Figure 1. The variables in the first three principal components (PCs) were highly correlated, with eigen values greater than 1 , thus explaining for $86.3 \%$ of the total variance, with PC1, PC2, and PC3 accounting for 45.9\%, 27.4\%, and 13.0\%, respectively. PC1 was positively correlated with $\mathrm{r}_{\mathrm{S}}$, LAA, TP, N, Cl and HAA; while it was negatively correlated with transpiration $(\mathrm{T}), \mathrm{A}_{\mathrm{CO} 2}$, dry biomass, Ca, nitrate, WUE, Mg and leaf area. Moreover, PC2 was positively correlated with $\mathrm{P}$, SPAD, fresh yield, LAA, leaf area and TP, while it was negatively correlated with Na, DM, and Cl.

The two cultivars were well separated but not univocally clustered in respect to PC1 and PC2. In fact, they changed in dependence on harvests/cuts along PC1 and in dependence on salinity concentration along PC2. In particular, red and green lettuce coming from cut 1 were distributed in the positive side of PC1, while green and red lettuce obtained at cut 2 were distributed in the negative side of PC1. Moreover, $1 \mathrm{mM} \mathrm{NaCl}$ was positioned in the positive side of PC2, while $30 \mathrm{mM} \mathrm{NaCl}$ was distributed in the negative side of PC2 (Figure 1). Interestingly, the combination red cultivar-cut 1 under 10 and $20 \mathrm{mM} \mathrm{NaCl}$, produced a higher premium quality lettuce (higher LAA, TP, HAA and $\mathrm{N})$. While the green cultivar-cut 2 under 1 and $10 \mathrm{mM} \mathrm{NaCl}$, accumulated more Ca and dry mass, but had also the highest contents of the antinutrient nitrate. Finally, in the higher left quadrant the red cultivar-cut 2 and green cultivar-cut 1, clustered together, showing lettuce characterized by the highest leaf area and fresh yield (Figure 1). 


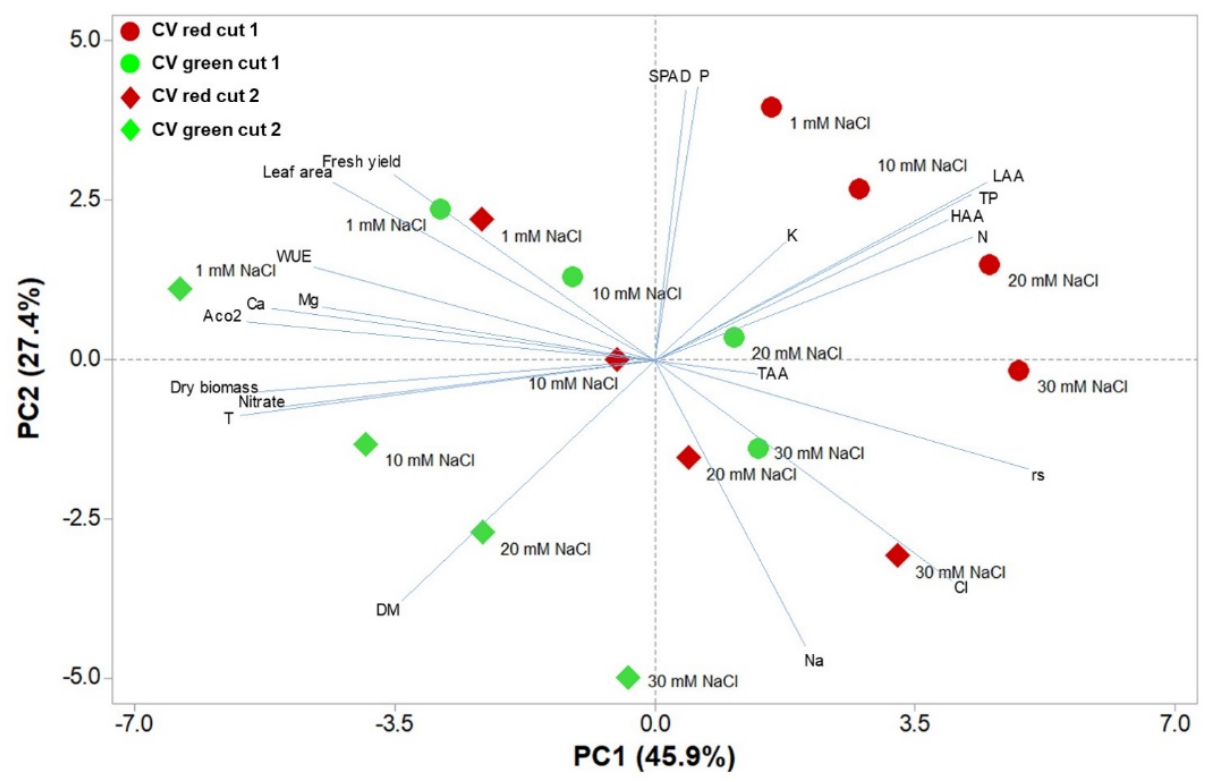

Figure 1. Principal component loading plot and scores of principal component analysis (PCA) of morphological parameters, mineral composition, leaf gas exchanges, bioactive compounds, and antioxidant activity of green and red pigmented lettuces under four salinity levels $(1,10,20$ and $30 \mathrm{mM} \mathrm{NaCl}$ ) and two successive harvests. T: transpiration rate, DM: lead dry matter percentage, TAA: total ascorbic acid, $\mathrm{r}_{\mathrm{s}}$ : stomatal resistance, HAA: hydrophilic antioxidant activity, LAA: Lipophilic antioxidant activity, SPAD: soil plant analysis development, WUE: water use efficiency, $\mathrm{A}_{\mathrm{CO} 2}$ : net $\mathrm{CO}_{2}$ assimilation rate.

\section{Discussion}

A mild stress (eustress) can induce plants to reshuffle plant metabolism, by triggering beneficial changes on nutritional and functional quality of the final products $[18,19,25]$. However, the increase of beneficial metabolites may affect plant growth and yield if the right amount of stress is not expertly administered to plants [18]. Therefore, in order to identify the best possible treatment to improve the nutritional quality of green and red baby lettuces without affecting productivity, we tried to understand how to modulate their physiological and biochemical parameters by using four salinity concentrations in combination with two successive harvests.

Green lettuce was significantly more productive than red one in terms of fresh yield, dry biomass, and leaf dry matter content, particularly at $1 \mathrm{mM} \mathrm{NaCl}$ salinity. This feature was certainly related to its lower stomatal resistance, which enhanced the net $\mathrm{CO}_{2}$ assimilation rate, notwithstanding the lower SPAD index present in the green cultivar, and consequently increased $\mathrm{WUE}_{\mathrm{i}}$, plant vegetative growth and productivity, as previously seen in green perilla [18]. This is in agreement with previous studies that showed that green lettuce cultivars generally grow faster than red ones [45]. However, green lettuce was more prone to the effect of salinity than the other cultivar, despite neither green lettuce nor red one were able to retain lower contents of $\mathrm{Na}$, and in addition, the red cultivar significantly accumulated more $\mathrm{Cl}$ than the green one, and this increase was even higher at cut 2 . $\mathrm{Na}$ and $\mathrm{Cl}$ are both toxic when present at high concentrations in cytosol and organelles [46]; however, Na toxicity does not depend on its absolute amount in the cytosol, but mainly on the $\mathrm{K}$ to $\mathrm{Na}$ ratio present in the cell and on the capacity of cell to compartmentalize this ion in the vacuole [47-49]. Indeed, the fact that only in the green cultivar, $\mathrm{K}$ decreased as the salinity in the nutrient solution increased, could certainly account for the higher sensitivity of this cultivar to salinity. Whereas, the ability of red lettuce to retain $\mathrm{K}$ at a constant level, independently of salinity, contributed to maintain higher K to Na ratio in the cytoplasm than green one, eventually also using stored $\mathrm{K}$ in the vacuole [46]. This latter mechanism could allow red lettuce to satisfy plant metabolic demand for $\mathrm{K}$ under salinity, by compartmentalizing the majority of $\mathrm{K}$ in the cytosol and $\mathrm{Na}$ in the vacuole [50]. It has been proved that even low concentrations of $\mathrm{K}$ 
exclusively compartmentalized in the cytosol, which accounts for less than $10 \%$ of the cell volume, may generate a remarkable osmotic pressure able to counteract the osmotic potential of vacuole, minimizing stress damage and re-establishing growth [47,51].

On the contrary, in green lettuce, as salinity increased, $\mathrm{K}$ decreased making probably $\mathrm{Na}$ accumulating at higher concentration in the cytosol. This event probably determined an alteration in the uptake and translocation of $\mathrm{K}$, which further decreased, and bivalent cations $\mathrm{Ca}$ and $\mathrm{Mg}$, which decreased more in green than in red lettuce at 20 and $30 \mathrm{mM} \mathrm{NaCl}$, due to the competitive interactions with $\mathrm{Na}$ [52]. Therefore, green but also red lettuce even at a lesser degree, became more sensitive to $\mathrm{Na}$ and/or $\mathrm{Cl}$ damages at 20 and $30 \mathrm{mM} \mathrm{NaCl}$, resulting in biomass reduction and fresh yield loss.

To the decrease of these macro-nutrients could be indirectly ascribed the more pronounced increase of stomatal resistance and decrease of photosynthetic rate present in green lettuce in comparison to the other cultivar. The present study revealed that salinity treatment reduced stomatal conductance and, consequently, photosynthetic rate in lettuce because of a reduction in macro-nutrients. Whereas, in Amaranthus leafy vegetables a stress dependent reduction in photosynthetic efficiency was demonstrated due to the reduction in photosynthetic pigments [53-56]. In agreement with our results, Ridolfi et al. [57] demonstrated that a decrease of Ca concentration could affect stomatal opening during dark-light transitions, decreasing stomatal conductance under full light and net $\mathrm{CO}_{2}$ assimilation rates. In addition, $\mathrm{K}$ is the main inorganic osmolyte modulating the guard cell turgor and stomatal movement, and its decrease exerts a negative influence on these physiological functions [58]. Moreover, since $\mathrm{Mg}$ is involved in the activation of $\mathrm{RuBisCO}$, via carbamylation, and other redox regulated enzymes, its decrease may reduce the rate of Calvin cycle, consequently slowing down the rate of photosynthetic electron transport chain, therefore contributing to reduce photosynthetic rate and generating photo-oxidation [58]. Mg decrease can also affect the phloem export of sucrose from source leaves impairing photoassimilates partitioning between shoots and roots and determining a relevant increase in the leaf dry matter due to the accumulation of starch and sucrose [59]. The higher dry matter was in fact another effect of high $\mathrm{NaCl}$ salinity on green lettuce.

However, previous studies have demonstrated that salinity tolerance varies with cultivars/genotypes not only in dependence on the capacity of plants to exclude and/or compartmentalize toxic ions, but also on the ability to enact responses aimed at minimizing stress damages and re-establishing biochemical and physiological functions and, therefore growth [19,60-62]. In this view, the higher contents of LAA and antioxidant agents such as TP and TAA present in red than in green baby lettuce were genetically determined, and their increase at the onset of stress (starting from $10 \mathrm{mM} \mathrm{NaCl}$ ) favored the tolerance response of red lettuce to stress. In addition, the increase of phenolics, among which the largest group is that of flavonoids, is also responsible for the leaf color of red lettuce that strongly influence consumer preference and choice making $[2,10,18,25]$.

At cut 2 both cultivars were able to accumulate more $\mathrm{Ca}$ and $\mathrm{Mg}$ than at cut 1 except that at $30 \mathrm{mM} \mathrm{NaCl}$, and only the green cultivar accumulated more $\mathrm{K}$. The increase in macro-minerals can be associated with health-promoting functions because $\mathrm{K}$ is able to lower blood pressure and favor elimination of toxins, while $\mathrm{Ca}$ and $\mathrm{Mg}$ may contribute to improved skeletal health and prevent osteoporosis [63]. Moreover, this increase of macro-nutrients could be associated with the decreased $r_{s}$, slight increase in photosynthetic rate as well as dry biomass and leaf dry matter content in both cultivars, but neither $\mathrm{WUE}_{\mathrm{i}}$ nor fresh weight or antioxidant activity increased, with the exception of TAA in red lettuce, compared to cut 1 . Accordingly, Corrado et al. [64] found that both the lipid-soluble and water-soluble antioxidant activity of basil leaves from the second cut were $15 \%$ lower than those in leaves of the first cut. At cut 2 the photosynthates in lettuce plants were probably mostly used for increasing the number of leaves and root area (not shown), in addition to the leaf area indirectly enhancing transpiration and uptake of nutrients, among which not only the beneficial $\mathrm{K}, \mathrm{Ca}$ and $\mathrm{Mg}$ but also the antinutrient nitrate, in particular in green lettuce, while the total $\mathrm{N}$ decreased. Anyway, the nitrate content remained within the limits established by the market according to EU regulation no. 1258/2011 (e.g., lower than $3000-5000 \mathrm{mg} \mathrm{kg}^{-1} \mathrm{fw}$ ). Accordingly, Corrado et al. [64] 
found higher concentration of nitrate in basil leaves at the second cut but also a higher total nitrogen content. This means that lettuce plants were efficient in uptaking and transporting nitrate to leaves, although the nitrate assimilatory reducing pathway was not. This higher accumulation of nitrate in lettuce leaves at second harvest happened notwithstanding the hydroponically grown plants were supplied with equal and constant amount of nitrogen fertilization until the end of cultivation. This phenomenon can directly depend on the higher levels of Mg and ATP (probably produced by the higher photosynthetic activity) present in lettuce tissues at the second harvest. In fact, nitrate reductase (NR), the enzyme catalyzing the assimilatory reduction of nitrate into nitrite [65], undergoes a reversible protein phosphorylation on a serine residue in presence of $\mathrm{Mg}$ and ATP; the phosphorylated enzyme binds to 14-3-3 proteins, inhibiting enzyme activity and making it more susceptible to proteolytic degradation [66]. Such inhibiting effect of Mg and ATP on NR is widespread among higher plants [67], and could be therefore responsible for the higher accumulation of nitrate in both lettuce varieties at the second harvest. It is important to underline that in these two varieties of lettuce the positive effect of salinity on mitigating the accumulation of nitrate was evident only under $30 \mathrm{mM} \mathrm{NaCl}$, a salinity concentration that highly affected plant fresh yield. Therefore, in this case the mechanism behind the decrease of nitrate more than an uptake inhibition due to the competition between $\mathrm{NO}_{3}{ }^{-}$and $\mathrm{Cl}^{-}$[68], could be the reduction in plant growth and development, causing a down regulation of net nitrate uptake $[35,69]$.

\section{Conclusions}

Salinity eustress in combination with successive harvests modulated the physiological and chemical composition of red and green pigmented genotypes of baby lettuce grown in floating raft system. Our study demonstrated the differential response of lettuce genotypes to salinity stress, with the green cultivar exhibiting higher sensitivity to salinity. Moreover, it was clearly shown that mild salinity can induce an increase of phytonutrients (total phenols and total ascorbic acid) and beneficial minerals $(\mathrm{K}, \mathrm{Ca}$, and $\mathrm{Mg})$ in red lettuce, thus enhancing the nutraceutical and nutritional quality of the product. This improvement was incurred at the expense of an acceptable, moderate yield reduction, ranging between $8 \%$ and $10 \%$ at 10 and $20 \mathrm{mM} \mathrm{NaCl}$, respectively. In particular, the increase in antioxidant metabolites in this cultivar was noteworthy since phenolics and ascorbic acid may contribute to the extension of the product's shelf-life and underpin its nutraceutical value by imparting anti-oxidant, anti-inflammatory and anti-proliferative properties, essential to prevent or treat cancer, cardiovascular and neurodegenerative diseases. As discussed above, the increase in macro-minerals can been associated also with pivotal health-promoting functions. Our results additionally demonstrated an improved leaf mineral status $(\mathrm{K}, \mathrm{Ca}$, and $\mathrm{Mg}$ ) recorded in lettuce plants coming from the second cut, irrespective of the salt stress treatment and genotype effects. Total ascorbic acid content in red lettuce increased at cut 2 whereas in green lettuce it was unaltered. Finally, these findings suggest that salinity eustress is a simple tool to exploit the quantitative variability of selected genotypes and could be valuable for accelerating breeding programs.

Author Contributions: Conceptualization, Y.R.; methodology, Y.R.; software M.G., G.R., F.N., and E.D.S.; validation M.G., G.R., F.N., and E.D.S.; formal analysis M.G., G.R., F.N., and E.D.S.; investigation M.I.S. and Y.R.; resources M.I.S. and Y.R.; data curation M.G., G.R., F.N., and E.D.S.; writing-original draft preparation P.C. and Y.R.; writing-review and editing, P.C. and Y.R.; visualization M.C.K. and M.I.S.; supervision Y.R.; project administration, Y.R.; funding acquisition M.I.S. and Y.R. All authors have read and agreed to the published version of the manuscript.

Funding: This research received no external funding.

Acknowledgments: The authors are grateful to Christophe El-Nakhel for reading the final draft and Antonio Pannico for the statistical analysis.

Conflicts of Interest: The authors declare no conflict of interest. 


\section{References}

1. Sofo, A.; Lundegårdh, B.; Mårtensson, A.; Manfra, M.; Pepe, G.; Sommella, E.; De Nisco, M.; Tenore, G.C.; Campiglia, P.; Scopa, A. Different agronomic and fertilization systems affect polyphenolic profile, antioxidant capacity and mineral composition of lettuce. Sci. Hortic. 2016, 204, 106-115. [CrossRef]

2. Alvino, A.; Barbieri, G. Vegetables of temperate climates: Leafy vegetables. In The Encyclopedia of Food and Health; Oxford Academic Press: Oxford, UK, 2016; pp. 393-400. [CrossRef]

3. Pepe, G.; Sommella, E.; Manfra, M.; De Nisco, M.; Tenore, G.C.; Scopa, A.; Sofo, A.; Marzocco, S.; Adesso, S.; Novellino, T.; et al. Evaluation of anti-inflammatory activity and fast UHPLC-DAD-IT-TOF profiling of polyphenolic compounds extracted from green lettuce (Lactuca sativa L.; var. Maravilla de Verano). Food Chem. 2015, 167, 153-161. [CrossRef] [PubMed]

4. Rodríguez-Hidalgo, S.; Artés-Hernández, F.; Gómez, P.A.; Fernández, J.A.; Artés, F. Quality of fresh-cut baby spinach grown under a floating trays system as affected by nitrogen fertilisation and innovative packaging treatments. J. Sci. Food Agric. 2010, 90, 1089-1097. [CrossRef] [PubMed]

5. Gil, M.I.; Garrido, Y. Chapter 21.1-Leafy vegetables: Baby leaves. In Controlled and Modified Atmospheres for Fresh and Fresh-Cut Produce; Gil, M.I., Beaudry, R., Eds.; Academic Press: Cambridge, MA, USA, 2020; pp. 527-536. [CrossRef]

6. Massaglia, S.; Merlino, V.M.; Borra, D.; Bargetto, A.; Sottile, F.; Peano, C. Consumer attitudes and preference exploration towards fresh-cut salads using best-worst scaling and latent class analysis. Foods 2019, 8, 568. [CrossRef]

7. Llorach, R.; Martínez-Sánchez, A.; Tomás-Barberán, F.; Gil, M.; Ferreres, F. Characterisation of polyphenols and antioxidant properties of five lettuce varieties and escarole. Food Chem. 2008, 108, 1028-1038. [CrossRef]

8. Mulabagal, V.; Ngouajio, M.; Nair, A.; Zhang, Y.; Gottumukkala, A.; Nair, M. In vitro evaluation of red and green lettuce (Lactuca sativa) for functional food properties. Food Chem. 2010, 118, 300-306. [CrossRef]

9. Baslam, M.; Morales, F.; Garmendia, I.; Goicoechea, N. Nutritional quality of outer and inner leaves of green and red pigmented lettuces (Lactuca sativa L.) consumed as salads. Sci. Hortic. 2013, 151, 103-111. [CrossRef]

10. Kim, M.J.; Moon, Y.; Tou, J.C.; Mou, B.; Waterland, N.L. Nutritional value, bioactive compounds and health benefits of lettuce (Lactuca sativa L.). J. Food Compos. Anal. 2016, 49, 19-34. [CrossRef]

11. Durazzo, A.; Azzini, E.; Lazzé, M.; Raguzzini, A.; Pizzala, R.; Maiani, G.; Palomba, L. Antioxidants in Italian head lettuce (Lactuca sativa var. capitataL.) grown in organic and conventional systems under greenhouse conditions. J. Food Biochem. 2013, 38, 56-61. [CrossRef]

12. Ribas-Agustí, A.; Gratacós-Cubarsí, M.; Sárraga, C.; García-Regueiro, J.-A.; Castellari, M. Analysis of eleven phenolic compounds including novel p-coumaroyl derivatives in lettuce (Lactuca sativa L.) by ultra-high-performance liquid chromatography with photodiode array and mass spectrometry detection. Phytochem. Anal. 2011, 22, 555-563. [CrossRef]

13. Shahidi, F.; Ambigaipalan, P. Phenolics and polyphenolics in foods, beverages and spices: Antioxidant activity and health effects-A review. J. Funct. Foods 2015, 18, 820-897. [CrossRef]

14. Złotek, U.; Świeca, M.; Jakubczyk, A. Effect of abiotic elicitation on main health-promoting compounds, antioxidant activity and commercial quality of butter lettuce (Lactuca sativa L.). Food Chem. 2014, 148, 253-260. [CrossRef] [PubMed]

15. Kim, H.-J.; Fonseca, J.M.; Choi, J.-H.; Kubota, C.; Kwon, D.Y. Salt in irrigation water affects the nutritional and visual properties of romaine lettuce (Lactuca sativa L.). J. Agric. Food Chem. 2008, 56, 3772-3776. [CrossRef] [PubMed]

16. Annunziata, M.G.; Attico, A.; Woodrow, P.; Oliva, M.A.; Fuggi, A.; Carillo, P. An improved fluorimetric HPLC method for quantifying tocopherols in Brassica rapa L. subsp. sylvestris after harvest. J. Food Compos. Anal. 2012, 27, 145-150. [CrossRef]

17. Sarno, V.; Manzo, R. Italian companies' attitude towards GM crops. Nutr. Food Sci. 2016, 46, 685-694. [CrossRef]

18. Rouphael, Y.; Kyriacou, M.C.; Carillo, P.; Pizzolongo, F.; Romano, R.; Sifola, M.I. Chemical eustress elicits tailored responses and enhances the functional quality of novel food perilla frutescens. Molecules 2019, 24, 185. [CrossRef]

19. Rouphael, Y.; Kyriacou, M.C. Enhancing quality of fresh vegetables through salinity eustress and biofortification applications facilitated by soilless cultivation. Front. Plant Sci. 2018, 9, 9. [CrossRef] 
20. Pérez-López, U.; Miranda-Apodaca, J.; Muñoz-Rueda, A.; Mena-Petite, A. Lettuce production and antioxidant capacity are differentially modified by salt stress and light intensity under ambient and elevated $\mathrm{CO}_{2}$. J. Plant Physiol. 2013, 170, 1517-1525. [CrossRef]

21. Pérez-López, U.; Miranda-Apodaca, J.; Lacuesta, M.; Mena-Petite, A.; Muñoz-Rueda, A. Growth and nutritional quality improvement in two differently pigmented lettuce cultivars grown under elevated $\mathrm{CO}_{2}$ and/or salinity. Sci. Hortic. 2015, 195, 56-66. [CrossRef]

22. Akula, R.; Ravishankar, G.A. Influence of abiotic stress signals on secondary metabolites in plants. Plant Signal. Behav. 2011, 6, 1720-1731. [CrossRef] [PubMed]

23. Woodrow, P.; Ciarmiello, L.F.; Annunziata, M.G.; Pacifico, S.; Iannuzzi, F.; Mirto, A.; D’Amelia, L.; Dell'Aversana, E.; Piccolella, S.; Fuggi, A.; et al. Durum wheat seedling responses to simultaneous high light and salinity involve a fine reconfiguration of amino acids and carbohydrate metabolism. Physiol. Plant. 2017, 159, 290-312. [CrossRef] [PubMed]

24. Lucini, L.; Borgognone, D.; Rouphael, Y.; Cardarelli, M.; Bernardi, J.; Colla, G. Mild Potassium chloride stress alters the mineral composition, hormone network, and phenolic profile in artichoke leaves. Front. Plant Sci. 2016, 7, 948. [CrossRef] [PubMed]

25. Kyriacou, M.C.; Rouphael, Y. Towards a new definition of quality for fresh fruits and vegetables. Sci. Hortic. 2018, 234, 463-469. [CrossRef]

26. Giordano, M.; El-Nakhel, C.; Pannico, A.; Kyriacou, M.C.; Stazi, S.R.; De Pascale, S.; Rouphael, Y. Iron biofortification of red and green pigmented lettuce in closed soilless cultivation impacts crop performance and modulates mineral and bioactive composition. Agronomy 2019, 9, 290. [CrossRef]

27. Petropoulos, S.A.; Levizou, E.; Ntatsi, G.; Fernandes, Â.; Petrotos, K.; Akoumianakis, K.; Barros, L.; Ferreira, I.C.F.R. Salinity effect on nutritional value, chemical composition and bioactive compounds content of Cichorium spinosum L. Food Chem. 2017, 214, 129-136. [CrossRef]

28. Neocleous, D.; Koukounaras, A.; Siomos, A.S.; Vasilakakis, M. Assessing the salinity effects on mineral composition and nutritional quality of green and red "Baby" lettuce. J. Food Qual. 2014, 37, 1-8. [CrossRef]

29. Sarker, U.; Islam, M.T.; Oba, S. Salinity stress accelerates nutrients, dietary fiber, minerals, phytochemicals and antioxidant activity in Amaranthus tricolor leaves. PLOS ONE 2018, 13, e0206388. [CrossRef]

30. Sarker, U.; Oba, S. Salinity stress enhances color parameters, bioactive leaf pigments, vitamins, polyphenols, flavonoids and antioxidant activity in selected Amaranthus leafy vegetables. J. Sci. Food Agric. 2019, 99, 2275-2284. [CrossRef]

31. Sarker, U.; Oba, S. Augmentation of leaf color parameters, pigments, vitamins, phenolic acids, flavonoids and antioxidant activity in selected Amaranthus tricolor under salinity stress. Sci. Rep. 2018, 8, 12349. [CrossRef]

32. Colla, G.; Rouphael, Y.; Cardarelli, M.; Svecova, E.; Rea, E.; Lucini, L. Effects of saline stress on mineral composition, phenolic acids and flavonoids in leaves of artichoke and cardoon genotypes grown in floating system. J. Sci. Food Agric. 2013, 93, 1119-1127. [CrossRef]

33. Rouphael, Y.; Cardarelli, M.; Lucini, L.; Rea, E.; Colla, G. Nutrient solution concentration affects growth, mineral composition, phenolic acids, and flavonoids in leaves of artichoke and cardoon. HortScience 2012, 47, 1424-1429. [CrossRef]

34. Tomasi, N.; Pinton, R.; Costa, L.D.; Cortella, G.; Terzano, R.; Mimmo, T.; Scampicchio, M.; Cesco, S. New 'solutions' for floating cultivation system of ready-to-eat salad: A review. Trends Food Sci. Technol. 2015, 46, 267-276. [CrossRef]

35. Borgognone, D.; Rouphael, Y.; Cardarelli, M.; Lucini, L.; Colla, G. Changes in biomass, mineral composition, and quality of cardoon in response to $\mathrm{NO}_{3}{ }^{-}: \mathrm{Cl}^{-}$ratio and nitrate deprivation from the nutrient solution. Front. Plant Sci. 2016, 7, 978. [CrossRef] [PubMed]

36. Barbieri, G.; Bottino, A.; Di Stasio, E.; Vallone, S.; Maggio, A. Proline and light as quality enhancers of rocket (Eruca sativa Miller) grown under saline conditions. Sci. Hortic. 2011, 128, 393-400. [CrossRef]

37. Nicoletto, C.; Santagata, S.; Bona, S.; Sambo, P. Influence of cut number on qualitative traits in different cultivars of sweet basil. Ind. Crops Prod. 2013, 44, 465-472. [CrossRef]

38. Sarker, U.; Hossain, M.M.; Oba, S. Nutritional and antioxidant components and antioxidant capacity in green morph Amaranthus leafy vegetable. Sci. Rep. 2020, 10, 1336. [CrossRef]

39. Rouphael, Y.; Colla, G.; Graziani, G.; Ritieni, A.; Cardarelli, M.; De Pascale, S. Phenolic composition, antioxidant activity and mineral profile in two seed-propagated artichoke cultivars as affected by microbial inoculants and planting time. Food Chem. 2017, 234, 10-19. [CrossRef] 
40. Sarker, U.; Oba, S.; Daramy, M.A. Nutrients, minerals, antioxidant pigments and phytochemicals, and antioxidant capacity of the leaves of stem amaranth. Sci. Rep. 2020, 10, 3892. [CrossRef] [PubMed]

41. Sarker, U.; Oba, S. Nutrients, minerals, pigments, phytochemicals, and radical scavenging activity in Amaranthus blitum leafy vegetables. Sci. Rep. 2020, 10, 3868. [CrossRef]

42. Fogliano, V.; Verde, V.; Randazzo, G.; Ritieni, A. Method for measuring antioxidant activity and its application to monitoring the antioxidant capacity of wines. J. Agric. Food Chem. 1999, 47, 1035-1040. [CrossRef]

43. Sarker, U.; Oba, S. Antioxidant constituents of three selected red and green color Amaranthus leafy vegetable. Sci. Rep. 2019, 9, 18233. [CrossRef] [PubMed]

44. Ciarmiello, L.F.; Piccirillo, P.; Carillo, P.; De Luca, A.; Woodrow, P. Determination of the genetic relatedness of fig (Ficus carica L.) accessions using RAPD fingerprint and their agro-morphological characterization. S. Afr. J. Bot. 2015, 97, 40-47. [CrossRef]

45. El-Nakhel, C.; Giordano, M.; Pannico, A.; Carillo, P.; Fusco, G.M.; De Pascale, S.; Rouphael, Y. Cultivar-specific performance and qualitative descriptors for butterhead salanova lettuce produced in closed soilless cultivation as a candidate salad crop for human life support in space. Life 2019, 9, 61. [CrossRef] [PubMed]

46. Carillo, P.; Cirillo, C.; De Micco, V.; Arena, C.; De Pascale, S.; Rouphael, Y. Morpho-anatomical, physiological and biochemical adaptive responses to saline water of Bougainvillea spectabilis Willd. trained to different canopy shapes. Agric. Water Manag. 2019, 212, 12-22. [CrossRef]

47. Annunziata, M.G.; Ciarmiello, L.F.; Woodrow, P.; Maximova, E.; Fuggi, A.; Carillo, P. Durum wheat roots adapt to salinity remodeling the cellular content of nitrogen metabolites and sucrose. Front. Plant Sci. 2017, 7, 515. [CrossRef]

48. Shabala, S.; Cuin, T.A. Potassium transport and plant salt tolerance. Physiol. Plant. 2008, 133, 651-669. [CrossRef]

49. Rouphael, Y.; Raimondi, G.; Lucini, L.; Carillo, P.; Kyriacou, M.C.; Colla, G.; Cirillo, V.; Pannico, A.; El-Nakhel, C.; De Pascale, S. Physiological and metabolic responses triggered by omeprazole improve tomato plant tolerance to $\mathrm{NaCl}$ stress. Front. Plant Sci. 2018, 9, 249. [CrossRef]

50. Wang, M.; Zheng, Q.; Shen, Q.; Guo, S. The critical role of potassium in plant stress response. Int. J. Mol. Sci. 2013, 14, 7370-7390. [CrossRef]

51. Cuin, T.A.; Tian, Y.; Betts, S.A.; Chalmandrier, R.; Shabala, S. Ionic relations and osmotic adjustment in durum and bread wheat under saline conditions. Funct. Plant Biol. 2009, 36, 1110-1119. [CrossRef]

52. Grieve, C.M.; Grattan, S. Mineral nutrient acquisition and response by plants grown in saline environments. In Handbook of Plant and Crop Stress; Marcel Dekker, Inc.: New York, NY, USA, 1999; pp. 203-229. [CrossRef]

53. Sarker, U.; Oba, S. Catalase, superoxide dismutase and ascorbate-glutathione cycle enzymes confer drought tolerance of Amaranthus tricolor. Sci. Rep. 2018, 8, 16496. [CrossRef]

54. Sarker, U.; Oba, S. Drought stress effects on growth, ROS markers, compatible solutes, phenolics, flavonoids, and antioxidant activity in amaranthus tricolor. Appl. Biochem. Biotechnol. 2018, 186, 999-1016. [CrossRef] [PubMed]

55. Sarker, U.; Oba, S. Drought stress enhances nutritional and bioactive compounds, phenolic acids and antioxidant capacity of Amaranthus leafy vegetable. BMC Plant Biol. 2018, 18, 258. [CrossRef] [PubMed]

56. Sarker, U.; Oba, S. Response of nutrients, minerals, antioxidant leaf pigments, vitamins, polyphenol, flavonoid and antioxidant activity in selected vegetable amaranth under four soil water content. Food Chem. 2018, 252, 72-83. [CrossRef] [PubMed]

57. Ridolfi, M.; Roupsard, O.; Garrec, J.; Dreyer, E. Effects of a calcium deficiency on stomatal conductance and photosynthetic activity of Quercus robur seedlings grown on nutrient solution. Ann. For. Sci. 1996, 53, 325-335. [CrossRef]

58. Tränkner, M.; Tavakol, E.; Jákli, B. Functioning of potassium and magnesium in photosynthesis, photosynthate translocation and photoprotection. Physiol. Plant. 2018, 163, 414-431. [CrossRef]

59. Cakmak, I.; Kirkby, E.A. Role of magnesium in carbon partitioning and alleviating photooxidative damage. Physiol. Plant 2008, 133, 692-704. [CrossRef]

60. Rouphael, Y.; Kyriacou, M.C.; Petropoulos, S.A.; De Pascale, S.; Colla, G. Improving vegetable quality in controlled environments. Sci. Hortic. 2018, 234, 275-289. [CrossRef]

61. Ferchichi, S.; Hessini, K.; Dell'Aversana, E.; D'Amelia, L.; Woodrow, P.; Ciarmiello, L.F.; Fuggi, A.; Carillo, P. Hordeum vulgare and Hordeum maritimum respond to extended salinity stress displaying different temporal accumulation pattern of metabolites. Funct. Plant Biol. 2018, 45, 1096-1109. [CrossRef] 
62. Song, Y.; Nakajima, T.; Xu, D.; Homma, K.; Kokubun, M. Genotypic variation in salinity tolerance and its association with nodulation and nitrogen uptake in soybean. Plant Prod. Sci. 2017, 20, 490-498. [CrossRef]

63. Gharibzahedi, S.M.T.; Jafari, S.M. The importance of minerals in human nutrition: Bioavailability, food fortification, processing effects and nanoencapsulation. Trends in Food Sci. Technol. 2017, 62, 119-132. [CrossRef]

64. Corrado, G.; Chiaiese, P.; Lucini, L.; Miras-Moreno, B.; Colla, G.; Rouphael, Y. Successive harvests affect yield, quality and metabolic profile of sweet basil (Ocimum basilicum L.). Agronomy 2020, 10, 830. [CrossRef]

65. Van Oosten, M.J.; Dell'Aversana, E.; Ruggiero, A.; Cirillo, V.; Gibon, Y.; Woodrow, P.; Maggio, A.; Carillo, P. Omeprazole treatment enhances nitrogen use efficiency through increased nitrogen uptake and assimilation in corn. Front. Plant Sci. 2019, 10, 10. [CrossRef] [PubMed]

66. Carillo, P.; Mastrolonardo, G.; Nacca, F.; Fuggi, A. Nitrate reductase in durum wheat seedlings as affected by nitrate nutrition and salinity. Funct. Plant Biol. 2005, 32, 209-219. [CrossRef] [PubMed]

67. Lillo, C.; Smith, L.H.; Nimmo, H.G.; Wilkins, M.B. Rhythms in magnesium ion inhibition and hysteretic properties of nitrate reductase in the CAM plant Bryophyllum fedtschenkoi. Physiol. Plant. 1996, 98, 140-146. [CrossRef]

68. Carillo, P.; Woodrow, P.; Raimondi, G.; El-Nakhel, C.; Pannico, A.; Kyriacou, M.C.; Colla, G.; Mori, M.; Giordano, M.; De Pascale, S.; et al. Omeprazole promotes chloride exclusion and induces salt tolerance in greenhouse basil. Agronomy 2019, 9, 355. [CrossRef]

69. Touraine, B.; Clarkson, D.T.; Muller, B. Regulation of nitrate uptake at the whole plant level. In A Whole Plant Perspective on Carbon-Nitrogen Interactions; SPB Academic Publishing: The Hague, The Netherlands, 1994; pp. 11-30.

(C) 2020 by the authors. Licensee MDPI, Basel, Switzerland. This article is an open access article distributed under the terms and conditions of the Creative Commons Attribution (CC BY) license (http://creativecommons.org/licenses/by/4.0/). 